\title{
Two-dimensional culture of human pancreatic adenocarcinoma cells results in an irreversible transition from epithelial to mesenchymal phenotype
}

\author{
Ya'an Kang ${ }^{1}$, Ran Zhang ${ }^{1}$, Rei Suzuki ${ }^{2}$, Shao-qiang Li ${ }^{3}$, David Roife ${ }^{1}$, Mark J Truty ${ }^{4}$, Deyali Chatterjee ${ }^{5}$, \\ Ryan M Thomas ${ }^{6}$, James Cardwell ${ }^{7}$, Yu Wang ${ }^{8}$, Huamin Wang ${ }^{9}$, Matthew H Katz ${ }^{1}$ and Jason B Fleming ${ }^{1}$
}

Many commercially available cell lines have been in culture for ages, acquiring phenotypes that differ from the original cancers from which these cell lines were derived. Therefore, research on new cell lines could improve the success rates of translational research in cancer. We have developed methods for the isolation and culture of human pancreatic ductal adenocarcinoma (PDAC) cells from murine xenografts of human PDAC. We hypothesize that phenotypes of PDAC cells are modified by in vitro culture conditions over time and by in vivo implantation. Patient-derived xenografts were created in immunodeficient mice using surgically resected tumor specimens. These murine xenografts were then used to establish human PDAC cell lines in culture. Earlier $(<5)$ passage and later $(>20)$ passage cell lines were evaluated separately regarding proliferation, cell cycle, genetic mutations, invasiveness, chemosensitivity, tumorigenesis, epithelialmesenchymal transition (EMT) status, and proteomics. Later passage cells accelerated their doubling time and colony formation, and were more concentrated in the G0/G1 phase and less in the G2/M checkpoint phase. Later passage cells were more sensitive to gemcitabine and 5-fluorouracil than earlier passage cells, but all four new cell lines were more chemo-resistant compared with commercial ATCC cell lines. EMT induction was observed when establishing and passaging cell lines in vitro and furthermore by growing them as subcutaneous tumors in vivo. This study demonstrates a novel approach to the establishment of PDAC cell lines and observes a process by which newly established cell lines undergo phenotypic changes during in vitro culture and in vivo tumorigenesis. This may help explain differences of treatment effects often observed between experiments conducted in vitro, in vivo, and in human clinical trials. Laboratory Investigation (2015) 95, 207-222; doi:10.1038/labinvest.2014.143; published online 8 December 2014

Pancreatic ductal adenocarcinoma (PDAC) is one of the most lethal malignancies. The American Cancer Society has estimated that in 2012, PDAC was diagnosed in 43392 Americans, and 37390 deaths were attributed to PDAC. Since 2004, the incidence of pancreatic cancer has been increasing by $1.2 \%$ per year. ${ }^{1}$ Despite extensive research, the prognosis has not significantly improved and the disease is almost uniformly lethal, with an average 5-year survival rate of less than $5 \%{ }^{2}$ One setback to research progress has been the limited amount of viable patient tumor-derived tissue.
Therefore, the majority of preclinical research in PDAC has been done in a limited number of commercially available cell lines. Publications from large studies have indicated that cell lines typically recapitulate the genomic events leading to neoplastic changes seen in patient samples. However, some genomic differences between tissues of origin and cell lines have been documented. ${ }^{3,4}$ Most commercial cell lines have grown for years in artificial culture conditions, and these long culture times render the cells prone to phenotypic and genetic drift. This is possibly why the majority of preclinical

\footnotetext{
'Department of Surgical Oncology, The University of Texas MD Anderson Cancer Center, Houston, TX, USA; ${ }^{2}$ Department of Gastroenterology and Rheumatology, The Fukushima Medical University School of Medicine, Fukushima, Japan; ${ }^{3}$ Department of Hepatobiliary Surgery, The First Affiliated Hospital of Sun Yat-sen University, Guangzhou, China; ${ }^{4}$ Department of Surgery, Mayo Clinic, Rochester, MN, USA; ${ }^{5}$ Department of Pathology and Immunology, Baylor College of Medicine, Houston, TX, USA; ${ }^{6}$ Department of General Surgery, The University of Florida College of Medicine, Gainesville, FL, USA; ${ }^{7}$ School of Medicine, Texas Tech Health Sciences Center, Lubbock, TX, USA; ${ }^{8}$ Neurodiagnostics Lab, The University of Texas Medical Branch, Galveston, TX, USA and ${ }^{9}$ Department of Pathology, The University of Texas MD Anderson Cancer Center, Houston, TX, USA

Correspondence: Professor JB Fleming, MD, Department of Surgical Oncology, Unit 0107, The University of Texas MD Anderson Cancer Center, 1515 Holcombe Blvd. Houston, TX 77030, USA.
}

E-mail: jbflemin@mdanderson.org

Received 14 July 2014; revised 3 September 2014; accepted 8 September 2014 
research fails to translate into successful human studies. To counter this, research laboratories should establish their own primary cell lines to obtain a greater heterogeneity of cell lines and to avoid the use of cell lines cultured for extended periods of time. Although various techniques have been used to isolate and culture human PDAC cells, cultivating and preserving these cells remains a challenge. ${ }^{4-6}$

In epithelial cell-derived cancers such as PDAC, numerous studies have shown that epithelial-mesenchymal transition (EMT) plays a central role in cancer progression and metastasis. However, there are little data regarding the status of this phenotypic change in cell line populations, specifically whether this phenotype differs between the original tumor tissue and the cell line that was derived from it. The initiation of metastasis requires cell invasion, which starts when tumor cells lose cell-cell adhesion mediated by E-cadherin downregulation. These cells break through the basement membrane with increased invasive properties, and then enter the bloodstream to form micrometastases. When these cells reach a distant site and implant, they undergo a reversal of this process, termed mesenchymal-epithelial transition (MET), and subsequently form clonal outgrowths at these metastatic sites. Thus, EMT and MET, respectively, comprise the initiation and completion of the invasion-metastasis cascade. $^{5}$ These $\mathrm{E}$ - and $\mathrm{N}$-cadherin proteins are calciumdependent cell-cell adhesion glycoproteins composed of five extracellular cadherin repeats, a transmembrane region, and a highly conserved cytoplasmic tail. ${ }^{6}$ Cadherin transitions between epithelial and mesenchymal states play important roles in embryonic development and cancer metastasis. An E-cadherin level change is considered to be a fundamental event in EMT and MET. Changes also occur in the expression of other cytoskeletal proteins, such as cytokeratin 19 and vimentin. ${ }^{7,8}$ Little is known about EMT-MET changes that occur when cell lines are transferred from in vivo to in vitro conditions, and vice-versa. Studies have suggested that repeated cycles of growing cancerous cell lines in nude mice cause these cell lines to become more aggressive. ${ }^{9-11}$ We hypothesize that this increase in aggressiveness is due to a transition from an epithelial to mesenchymal phenotype that occurs during cell line derivation and continues throughout cell culture.

In this study, we established four new PDAC cell lines from our patient-derived tumor xenograft (PATX) program $^{12}$ MDA-PATC43, MDA-PATC50, MDA-PATC53, and MDAPATC66. We analyzed these cell lines regarding proliferation, cell cycle, genetic mutations, chemosensitivity, invasiveness, tumorigenesis, EMT status, and proteomics. These data were obtained from cell lines separately in earlier $(<5)$ and later $(>20)$ cell passages in vitro. We identified the biologic discrepancies among PATX tumors and the PDAC cell lines derived from them. We also examined alterations in subcutaneous tumors created with these PDAC cells (termed sub-PATC) compared with the original PATX tumors and their derived cell lines.

\section{MATERIALS AND METHODS}

\section{PATX and Cell Culture Procedure}

Patient tumor specimens for PATX were obtained from primary pancreatic or metastatic liver tumor sites surgically. All patients were treated in the Department of Surgical Oncology at The University of Texas MD Anderson Cancer Center, and gave informed consent prior to surgery and tumor collection. The methods for developing and maintaining the PATX model have been previously reported. ${ }^{12}$ After the primary tumor was established in NOD/SCID mice, we harvested the tumor and divided it into separate portions for cell culture and for re-implantation into successive generations of NOD/ SCID mice. For the cell culture portion, we first removed the surrounding connective tissue and then washed the specimen with sterile phosphate-buffered saline containing $1 \%$ penicillin-streptomycin (Mediatech, Herndon, VA, USA). Then, using surgical scalpels, we finely minced the tumor tissue into cubes of approximately $1 \mathrm{~mm}^{3}$ in volume. No enzymatic degradation of the tumor tissues was performed in this procedure. The mechanical dissection method provided us an adequate number of cells for culture on dishes. The tissue was cultured in RPMI- 1640 with $2.5 \mathrm{mM} \mathrm{L-glutamine}$ (HyClone Laboratories, Logan, UT, USA) and 10\% US source fetal bovine serum (FBS; Mediatech). All media were supplemented with $1 \%$ penicillin and streptomycin. The cells were maintained at $37^{\circ} \mathrm{C}$ in a humidified atmosphere of $5 \% \mathrm{CO} 2$, and the medium was replaced every 3 days.

When cells outgrew from the tumor piece and formed well-isolated clones, we removed and discarded the growth medium, and washed dishes with phosphate-buffered saline twice. These individual clones were isolated via a cloning cylinder (Bel-Art Products, Wayne, NJ, USA). Selected clones were confirmed as human PDAC cells at the MD Anderson Molecular Cytogenetics core facility. New cell lines were further validated via short tandem repeat (STR) DNA fingerprinting using the AmpF/STR Identifier kit (Applied Biosystems cat 4322288) according to the manufacturer's instructions. The STR profiles were compared with known ATCC fingerprints (ATCC.org) and with the Cell Line Integrated Molecular Authentication database version 0.1.200808 (http://bioinformatics. istge.it/clima/) (Nucleic Acids Research 37:D925-D932 PMCID: PMC2686526). The results of the STR profile showed that our four new cell lines were unique. STR profiling did not detect any DNA other than human, and these cells showed an absence of mycoplasma through our core laboratory validation (MD Anderson Characterized Cell Line Core Facility). All experiments for this study were performed on cell lines in the fifth passage or later.

We also used the following commercial pancreatic cancer cell lines obtained from ATCC as controls: PANC-1 (ATCC, CRL-1469), MiaPaCa-2 (CRL-1420), and BxPC-3 (CRL-1687). All cell lines were grown in RPMI-1640 supplemented with $2 \mathrm{mM}$ L-glutamine, $1 \mathrm{mM}$ sodium pyruvate, $4.5 \mathrm{~g} / 1$ glucose, and 10\% FBS. All ATCC cell lines were cultured in a humidified atmosphere containing $5 \% \mathrm{CO} 2$ at $37^{\circ} \mathrm{C}$. 


\section{Genomic DNA and Total RNA Extraction}

Genomic DNA was extracted from freshly cultured cells using a Qiagen blood and cell culture DNA mini kit according to the manufacturer's instructions. DNA samples were analyzed for STR DNA fingerprinting and KRAS mutation status by the Sequencing and Microarray Core Facility at MD Anderson.

Total RNA was extracted using TRIzol (Life Technologies) and then purified via an RNeasy mini kit (Qiagen) according to the manufacturer's instructions. First-strand cDNA was synthesized using $1 \mu \mathrm{g}$ of total RNA with the SuperScript Mixture kit (Bio-Rad). The entire coding region of Smad4 and TP53 was amplified using PCR primers for every exon, as previously cited by Ding et al. and Liu et al. ${ }^{13,14}$ Smad4 and TP53 mutations were analyzed via direct sequencing of the PCR products by the Sequencing and Microarray Core Facility at MD Anderson. Smad4 immunohistochemical staining was performed on patient tumor slides and PATX tissue microarray slides to confirm Smad4 mutation status.

\section{Cell Doubling Times, Colony-Formation Assay, and Cell Cycle Analysis}

Cells proliferation was assessed via a standard MTT assay as previously described. ${ }^{15}$ A total of 1000 cells per well were seeded into a 96-well plate in triplicate. On days $1,3,5$, and 7, $20 \mu \mathrm{l}$ of a $5 \mathrm{mg} / \mathrm{ml}$ solution of MTT (Sigma-Aldrich, St Louis, MO, USA) was added to the wells and incubated for $3 \mathrm{~h}$ at $37^{\circ} \mathrm{C}$. The supernatant was removed, and the blue MTT formazan precipitates were then dissolved in $100 \mu \mathrm{l}$ dimethyl sulfoxide per well. The plates were placed on a shaker for $10 \mathrm{~min}$ at room temperature in the dark, and absorbance was measured on a plate reader at $570 \mathrm{~nm}$. Cell doubling times were generated via GraphPad Prism software (version 6.0 for Windows, GraphPad Software, La Jolla, CA, USA www. graphpad.com) according to their growth curves.

Colony-formation assays were performed by seeding $60 \mathrm{~mm}$ dishes in triplicate with 200 single cells of each of the four new PDAC cell lines. The colonies per dish were counted after 10 days in culture. Results were shown as the mean value \pm standard error using GraphPad Prism. The assay was repeated three times.

Cell cycle analysis was performed for earlier $(<5)$ and later $(>20)$ passages of each cell line. These cells were starved for $24 \mathrm{~h}$ in FBS-free media before cell cycle analysis, and then the cells were resuspended in $0.25 \%$ trypsin, washed twice with phosphate-buffered saline, and fixed in $70 \%$ ethanol. Cells were incubated in propidium iodide (Sigma-Aldrich) and triton X-100 staining solution with RNase A. As a control, these assays were also performed on the ATCC cell lines. Cell cycle profiles of these cells were analyzed by flow cytometry (BD Biosciences, San Jose, CA, USA). Cell cycle status was then analyzed with FlowJo software (www.flowjo.com). Each experiment was repeated three times. Results were expressed as the percentage of cells in each phase.

\section{Cytotoxicity Study}

The viability of each of these cell lines was evaluated during earlier $(<5)$ and later $(>20)$ passages using MTT assays. Cells were seeded in 96-well plates at a density of $3 \times 10^{3}$ cells/well in triplicate and allowed to attach overnight. The cells were then starved for $24 \mathrm{~h}$ with FBS-free medium prior to treatment. Cells were then treated with gemcitabine $(0.1 \mu \mathrm{M})$ and 5-fluorouracil $(500 \mu \mathrm{M})$ in RPMI 1640 with $10 \%$ FBS. After $72 \mathrm{~h}, 20 \mu \mathrm{l}$ of $5 \mathrm{mg} / \mathrm{ml} \mathrm{MTT} \mathrm{was} \mathrm{added} \mathrm{to}$ each well, and the cells were incubated for $3 \mathrm{~h}$ at $37^{\circ} \mathrm{C}$. The supernatants were discarded, $100 \mu \mathrm{l}$ dimethyl sulfoxide was added to each well, and the plates were incubated for $10 \mathrm{~min}$ at room temperature. The number of viable cells was determined by measuring the absorbance at $570 \mathrm{~nm}$ of insoluble formazan using a plate reader. This cytotoxicity study on early/late passage cell lines was also performed in Panc-1, BxPc3, and Miapaca2 cell lines as a control. All cells were incubated at $37^{\circ} \mathrm{C}$ in a humid atmosphere with $5 \% \mathrm{CO}_{2}$. Each experiment was repeated three times. Absorbance data were shown as the mean value \pm standard error of the three independent experiments by GraphPad Prism.

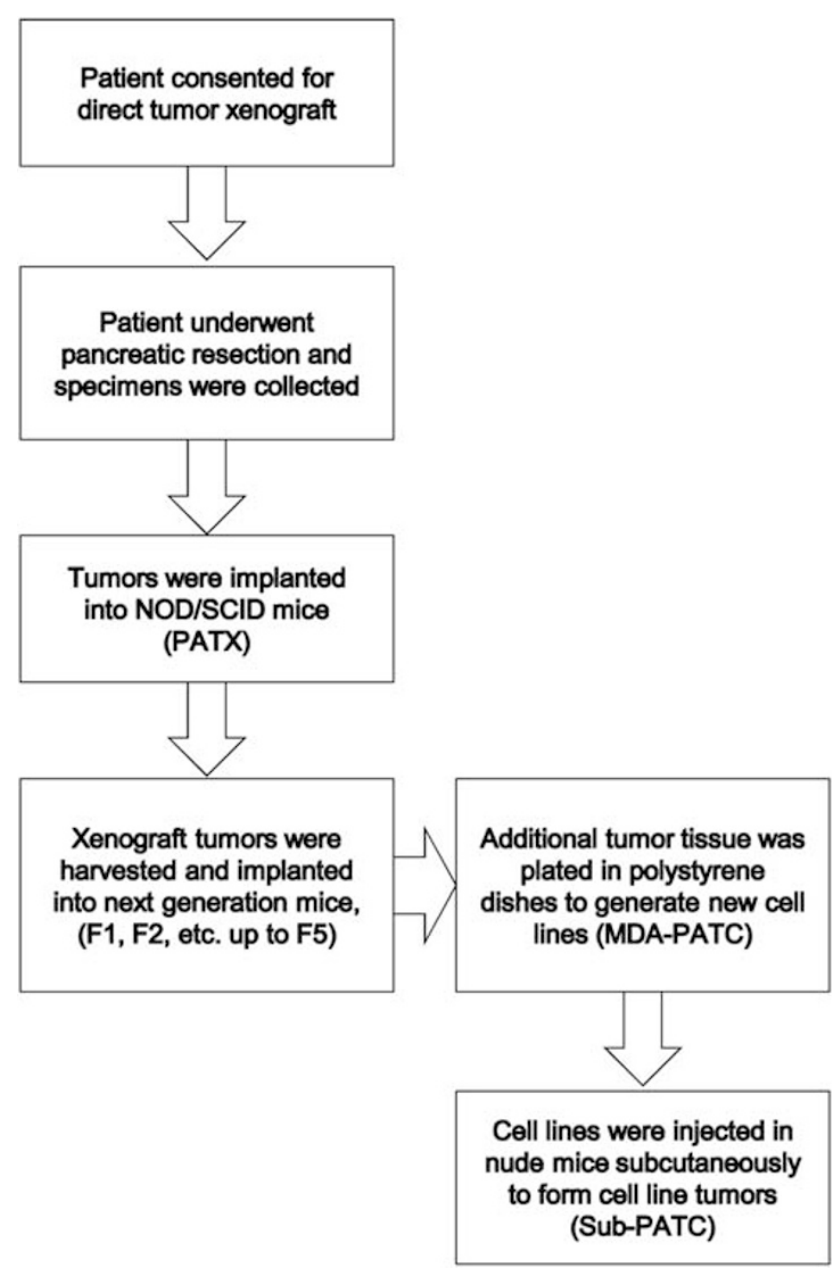

Figure 1 Summary of the methodological approach used for generating PDAC cell lines. 


\section{Western Blotting}

Sample lysates from these new cell lines, PATX tumors, and cell line subcutaneous tumors (Sub-PATCs) were lysed in RIPA buffer ( $50 \mathrm{mM}$ Tris $\mathrm{HCl}(\mathrm{pH}), 150 \mathrm{mM} \mathrm{NaCl}, 1 \%$ NP- $40,0.5 \%$ sodium deoxycholate, and $0.1 \%$ sodium dodecyl sulfate) supplemented with a protease inhibitor cocktail (Sigma-Aldrich). Proteins were electrophoresed in sample buffer on $8 \%-12 \%$ acrylamide gels and were then transferred to a polyvinylidene fluoride membrane (GE Healthcare, Piscataway, NJ, USA). To block nonspecific binding, the membrane was incubated for 1 or $2 \mathrm{~h}$ at room temperature in $0.5 \%$ TBST containing 5\% non-fat milk. The membranes were then probed with primary antibodies overnight at $4{ }^{\circ} \mathrm{C}$ on a rocker. The antibodies used were $\operatorname{Smad} 4(1: 1000)$ and $\beta$-catenin (1:1000) (Cell Signaling Technology, Boston, MA, USA), E-cadherin (1:5000), N-cadherin (1:2000), CK19 (1:1000), vimentin (1:1000), caveolin1 (1:1000), NF2 (1:8000), FoxM1 (1:500), and cyclin B1 (1:8000) (Abcam, Cambridge, MA, USA). TFRC $(1: 10,000)$ was purchased from Sigma (SigmaAldrich). $\beta$-actin (1:5000, Sigma) was used as protein loading control. The membranes were then probed with horseradish peroxidase-linked secondary anti-mouse or anti-rabbit antibodies (1:5000; Sigma-Aldrich) for $1 \mathrm{~h}$ at room temperature. After washing with TBST, the specified proteins were visualized using the ECL Western Blotting Detection System (GE Healthcare).

\section{Reverse Phase Protein Microarray}

The reverse phase protein microarray (RPPA) is a recently developed quantitative assay that analyzes nanoliter amounts of sample for potentially hundreds of proteins. RPPA is a high-throughput antibody-based technique developed for functional proteomics studies to evaluate protein activities in cell lines and patient tumor samples. ${ }^{16}$ This antibody-based assay determines levels of protein expression, protein modifications, and multiple signaling molecules and their functional status. The RPPA study has major strengths in identifying and validating cellular targets, characterizing signaling pathways and networks, and determining on- and off-target activities of novel drugs. ${ }^{16,17}$

RPPA analysis was performed in the Department of Systems Biology (Functional Proteomics Core Facility) at
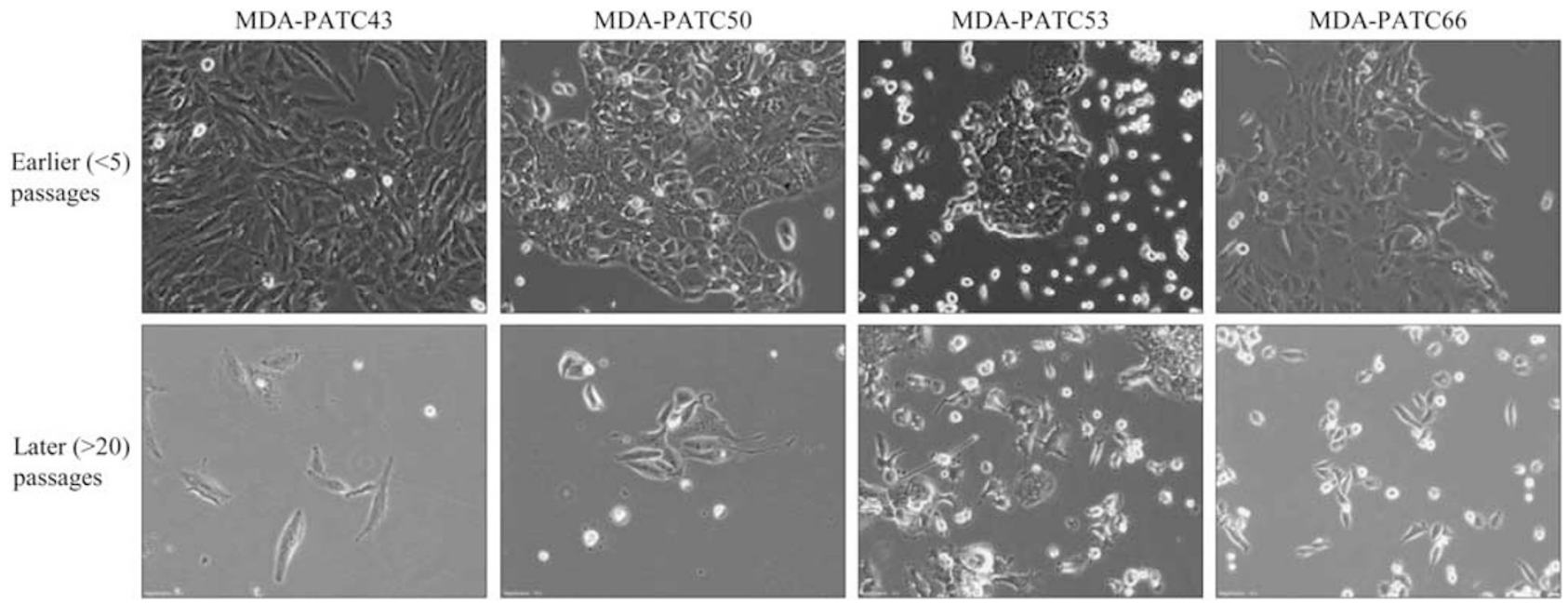

Figure 2 Morphology of new PDAC lines growing in culture. Top panel represents cells in earlier passages ( $<5$ passages), bottom panel shows cells grow pattern in later passages ( $>20$ passages). Magnification $\times 10$.

Table 1 Summary of the clinicopathological features of patients and gene statuses of derived cell lines ${ }^{a}$

\begin{tabular}{|c|c|c|c|c|c|c|c|}
\hline Cell lines & Sex & Age & Stage (TNM) & Tumor site & K-ras & $\mathrm{SMAD} 4^{\mathrm{b}}$ & TP53 \\
\hline MDA-PATC43 & $\mathrm{F}$ & 71 & 3.0 .0 & Pancreas & Cod 12: GGT;CGT & Delete & WT \\
\hline MDA-PATC50 & $\mathrm{F}$ & 60 & 3.1 .0 & Pancreas & Cod 12: GGT;CGT & Delete & $C(945,1061)>T$ \\
\hline MDA-PATC53 ${ }^{C}$ & $M$ & 65 & X.X.1 & Liver & Cod 12: GGT;GAT & $W^{d}$ & $W T^{d}$ \\
\hline MDA-PATC66 & $\mathrm{F}$ & 63 & 3.1 .0 & Pancreas & Cod 12: GGT; GAT & Delete & $\mathrm{T}(909,591)>\mathrm{A}$ \\
\hline
\end{tabular}

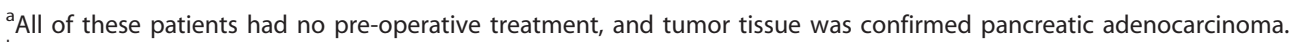

${ }^{b}$ Expression of SMAD4 protein was also demonstrated by immunohistochemistry in both patient specimens and murine xenograft tissue (Figure 3).

'Tumor from liver metastasis site.

dWild type. 
MD Anderson. To prepare lysates for RPPA analysis, PATX tumors, corresponding new cell lines, and sub-PATC tumors were lysed in lysis buffer ( $1 \%$ Triton X-100, $50 \mathrm{mM}$ HEPES ( $\mathrm{pH}$ 7.4), $150 \mathrm{mM} \mathrm{NaCl}, 1.5 \mathrm{mM} \mathrm{MgCl} 2,1 \mathrm{mM}$ EGTA, $100 \mathrm{mM} \mathrm{NaF}, 10 \mathrm{mM}$ Na pyrophosphate, $1 \mathrm{mM} \mathrm{Na} \mathrm{NO}_{3}$, $10 \%$ glycerol, containing freshly added protease and phosphatase inhibitors (Roche Applied Science, Indianapolis, IN, USA)). Sample proteins were denatured by $1 \%(\mathrm{w} / \mathrm{v})$ sodium dodecyl sulfate in the presence of $\beta$-mercaptoethanol and adjusted to a final concentration of $1 \mathrm{mg} / \mathrm{ml}$. Samples were diluted in five serial twofold dilutions in dilution buffer (lysis buffer containing $1 \%$ sodium dodecyl sulfate) and arrayed on nitrocellulose-coated slides (Grace Biolab) using an Aushon 2470 arrayer (Aushon BioSystems). Each slide was probed with a validated primary antibody plus a biotin-conjugated secondary antibody. The signal obtained was amplified using a

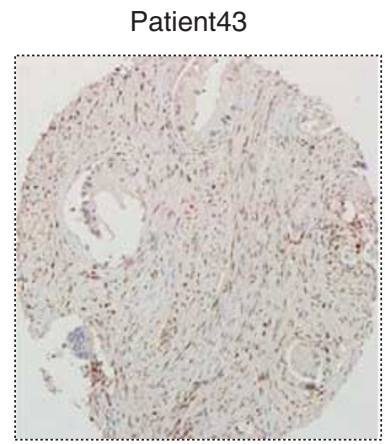

PATX43

b
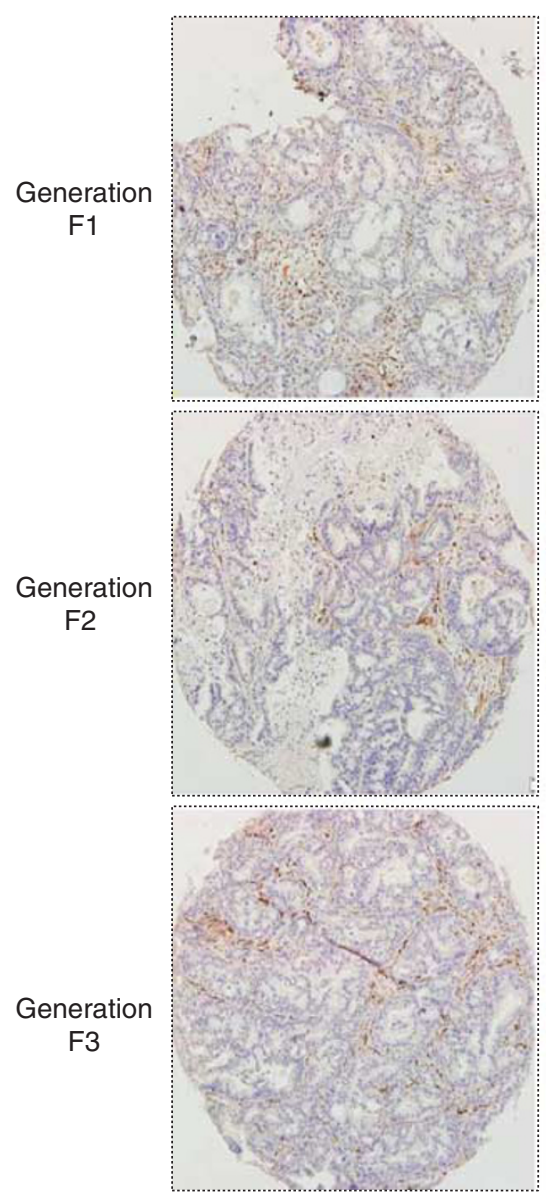

Patient50

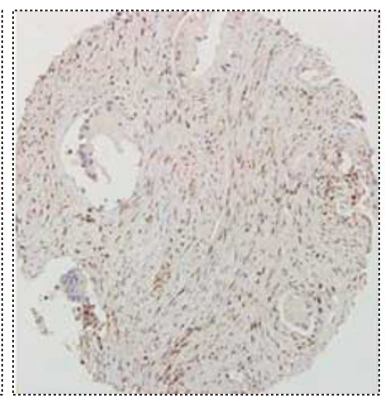

PATX50
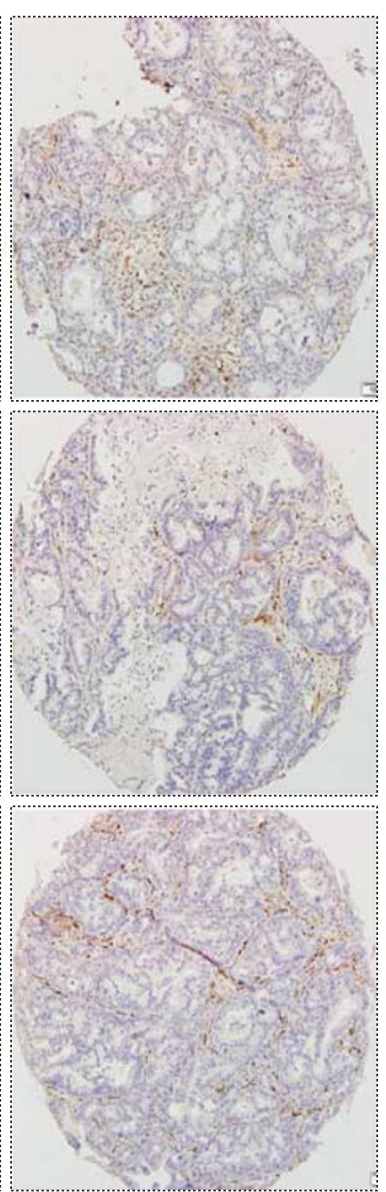

Patient53

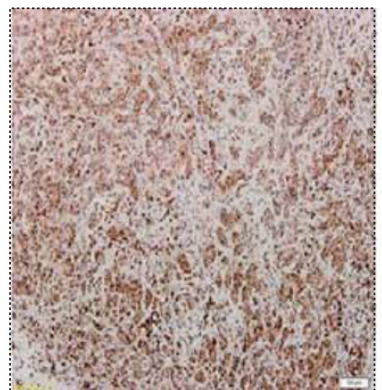

PATX53
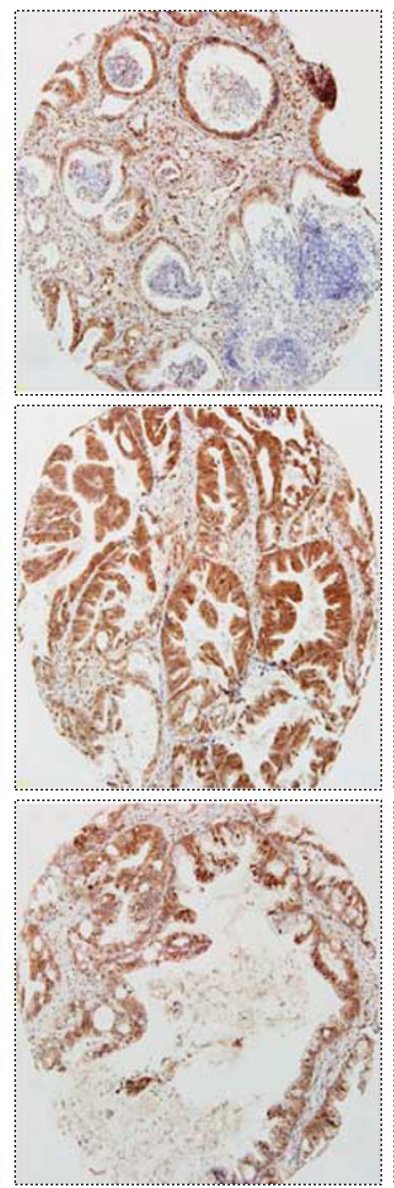

Patient66

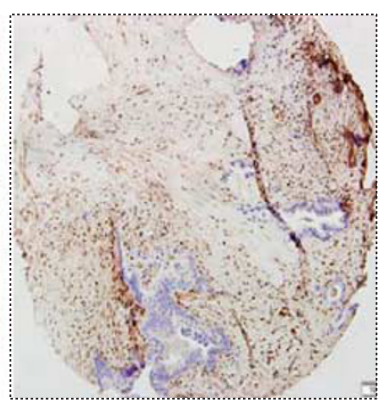

PATX66

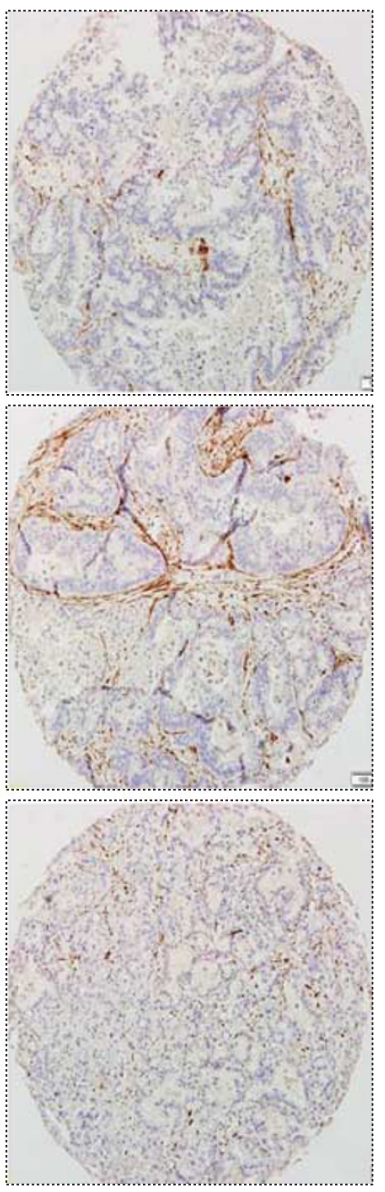

Figure 3 Immunohistochemical staining for SMAD4 was performed in (a) primary patient tumor tissue and (b) corresponding PATX tumors from generations F1, F2, and F3 in NOD/SCID mice. These were all stained concurrently in one tissue microarray slide. Staining was evaluated on ductal tumor cells. Patient 43,50, and 66 had a deletion of SMAD4, which is supported by negative staining of tumor cells in the original tumor and all three generations of murine xenografts. Patient 53 was wild type for SMAD4, represented by positive staining of ductal cells in the original tumor and all three generations of murine xenografts. See Table 1 for corresponding sequencing results. Magnification $\times 10$. 
a DakoCytomation-catalyzed system (Dako) and visualized via $\mathrm{DAB}$ colorimetric reaction. The slides were scanned, analyzed, and quantified using customized software, Microvigene (VigeneTech), to generate spot intensity. Each dilution curve was fitted with a logistic model ('Supercurve Fitting' developed by the Department of Bioinformatics and Computational Biology at MD Anderson Cancer Center; http:// bioinformatics.mdanderson.org/OOMPA) that fits a single curve using all the samples (eg, dilution series) on a slide with the signal intensity as the response variable and the dilution steps as independent variables. The fitted curve was plotted with the signal intensities-both observed and fitted-on the $y$-axis and the $\log 2$-concentration of proteins on the $x$-axis for diagnostic purposes. The protein concentrations of each set of slides were then normalized by median polish, which was corrected across samples by the linear expression values using the median expression levels of all antibody experiments to calculate a loading correction factor for each sample. The results for each antibody were then centered according to the mean, to facilitate visualization. A list of antibodies, including their sources and catalog numbers, may be found at: http:// www.mdanderson.org/education-and-research/resources-forprofessionals/scientific-resources/core-facilities-and-services/ functional-proteomics-rppa-core/antibodylists-protocols/ corestdablist-5-3-13.pdf. Unsupervised hierarchical clustering was performed using GENE-E (broadinstitute.org). The heatmap included was generated in Cluster 3.0 (http://www. eisenlab.org/eisen/) as a hierarchical cluster using Pearson
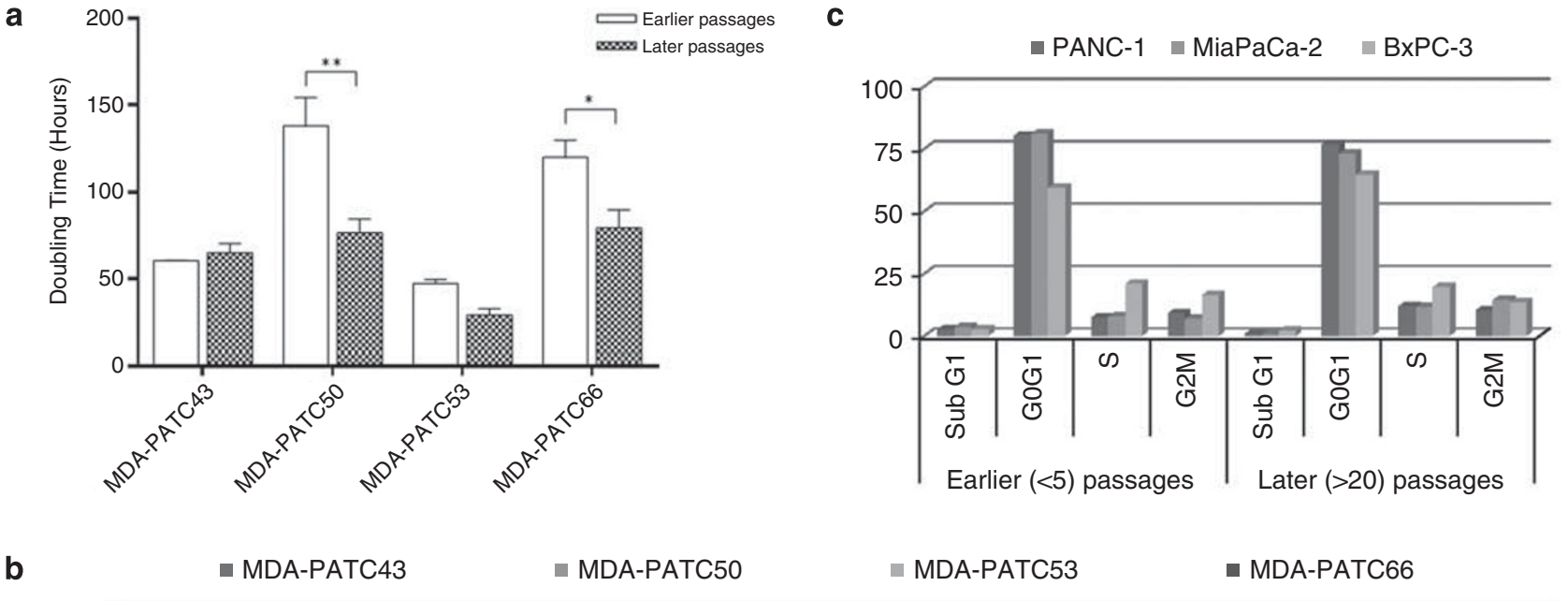

b MDA-PATC43 MDA-PATC50

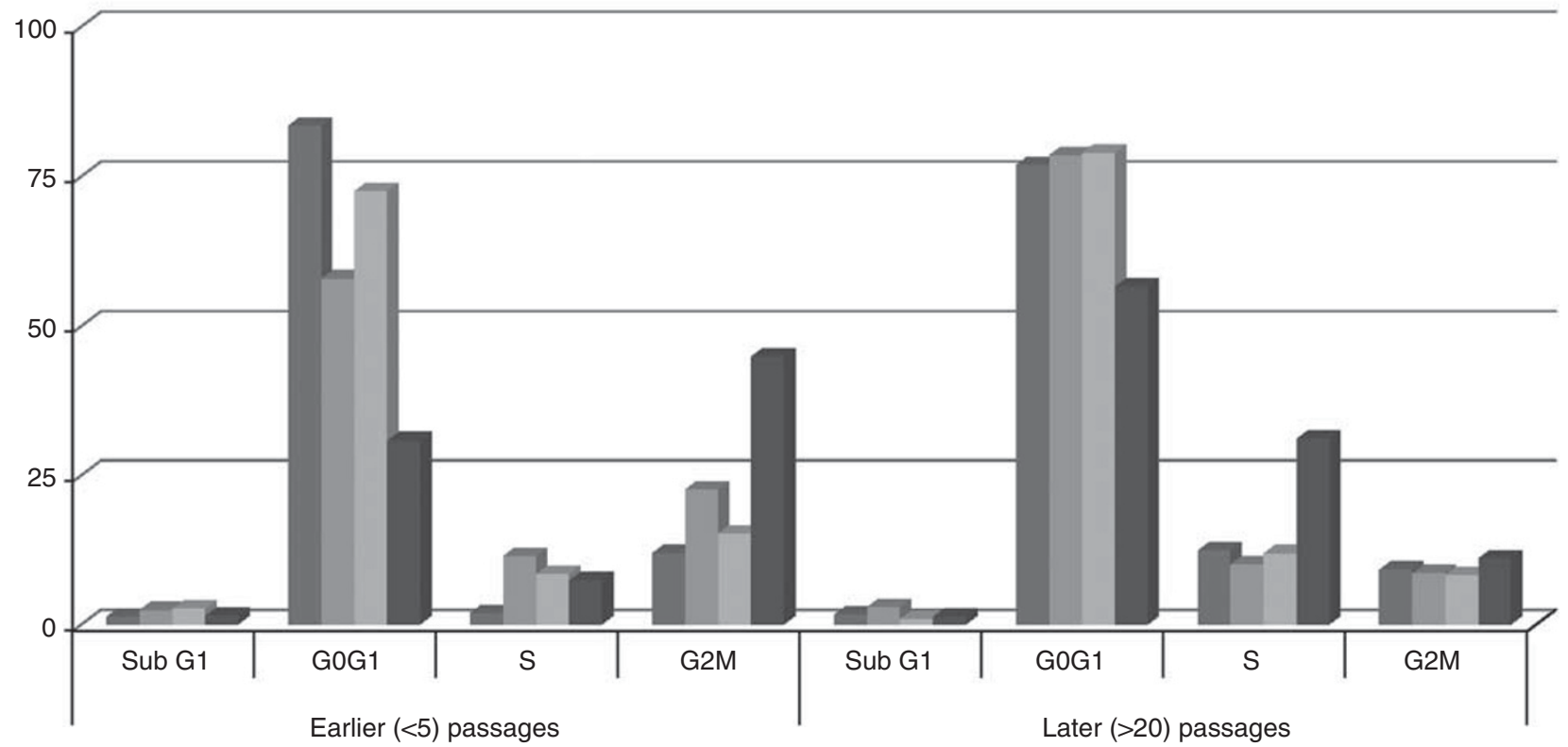

Figure 4 Comparison of MDA-PATC43, 50, 53, and 66 cell line proliferation between earlier and later passages. (a) Doubling time changes between earlier $(<5)$ and later $(>20)$ passages were assessed with serial MTT assays. Results are shown as means \pm s.d. and significance assessed via two-tailed $t$ test $\left({ }^{*} P<0.05\right.$ and $\left.{ }^{* *} P<0.01\right)$. (b) Flow cytometric analysis of cell cycle phase distribution comparing earlier and later passages in these four cell lines. (c) Flow cytometric cell cycle analysis of ATCC PDAC cell lines as a control. Cell cycle data collection and analysis were carried out by using CellQuest software. Assays were done thrice and in triplicate wells. The figure is a representative of three experiments with similar results. 
Correlation and a center metric. The resulting heatmap was visualized in Treeview (http://www.eisenlab.org/eisen/).

\section{In Vitro Invasive Capacity and In Vivo Tumor Growth Studies}

In vitro invasive capacity was measured using a BD-modified Boyden invasion chamber assay as previously described. ${ }^{18}$ These four cell lines were seeded in serum-free medium (RPMI) in the top compartment of matrigel-coated chambers $\left(5 \times 10^{4}\right.$ cells/chamber, $8.0-\mu \mathrm{m}$ pores, BD Biosciences, Bedford, MA, USA). RPMI $+10 \%$ FBS medium was placed in the bottom compartment as a chemoattractant. Cells were allowed to invade across the coated inserts for $20 \mathrm{~h}$. The cells on the apical surface of the insert were scraped off, and membranes containing invaded cells were fixed in $100 \%$ methanol, stained with $1 \%$ crystal violet (Sigma-Aldrich), and mounted on microscope slides. Invading cells were counted at $\times 10$ magnification in three different fields per membrane. Experiments were duplicated under each condition and repeated independently three times.

To evaluate the tumorgenicity of our four cell lines in vivo, we injected cells $\left(5 \times 10^{5}\right)$ from later passages of each cell line subcutaneously into the right flanks of three athymic nude mice per cell line. Cells were resuspended separately in sterile 50\% growth factor-reduced matrigel (BD Biosciences) in phosphatebuffered saline. The mice were monitored daily. Tumors were measured externally with calipers twice a week, and tumor volume was calculated using the following equation: volume $1 / 2$ $D \times d^{2}$, where $D$ is the long side and $d$ is the short side of the tumor. All groups of mice were killed at the 50th day, and their tumors were excised and weighed. Tumor tissues were processed for routine histologic and immunohistochemical analysis. The tumor volume grown curve and tumor weight comparison at day 50 were plotted by GraphPad Prism.

Animal studies were performed in accordance with the animal protocol procedures approved by the MD Anderson Cancer Center Institutional Review Board and Institutional Animal Care and Use Committee and were conducted in accordance with institutional and national regulations (protocol \# 01-11-00131).

\section{Immunohistochemical Staining}

Tissues from patients' primary tumors, PATX tumors, and subcutaneous cell line xenograft tumors were embedded in paraffin and then cut into $4 \mu \mathrm{m}$ thick sections for a
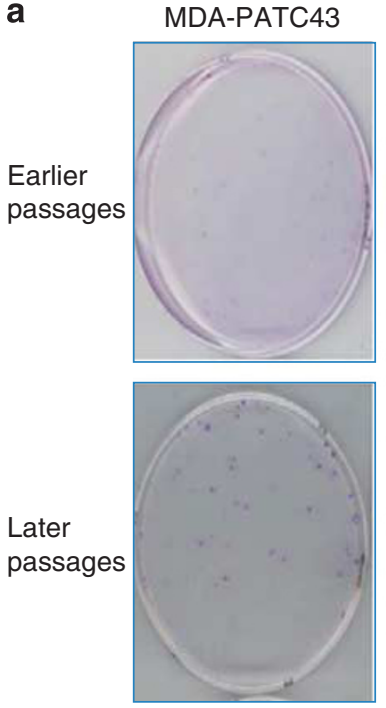
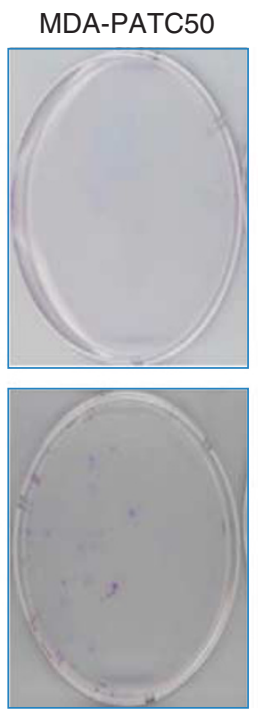
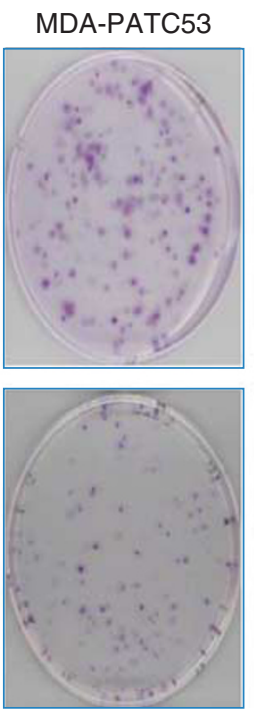

MDA-PATC66
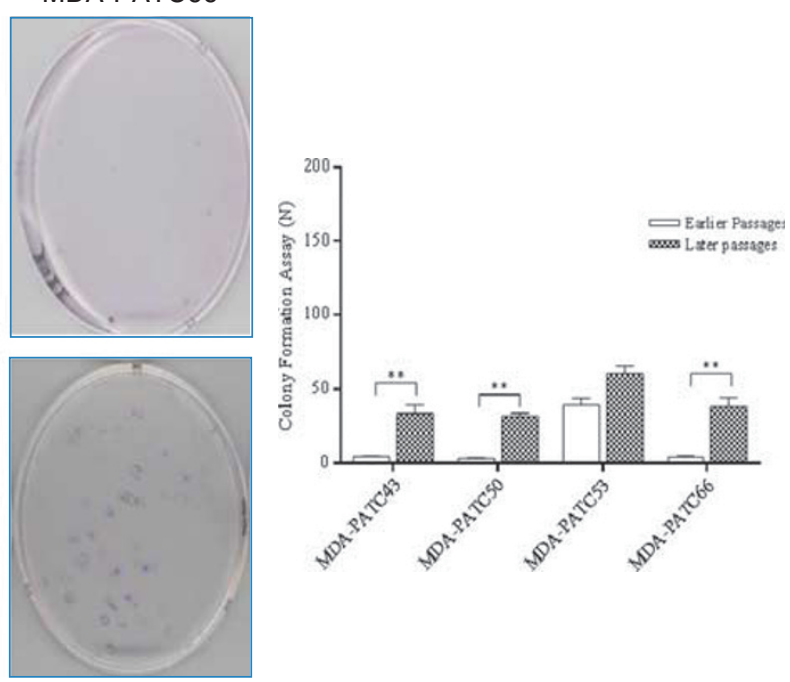

b PANC-1
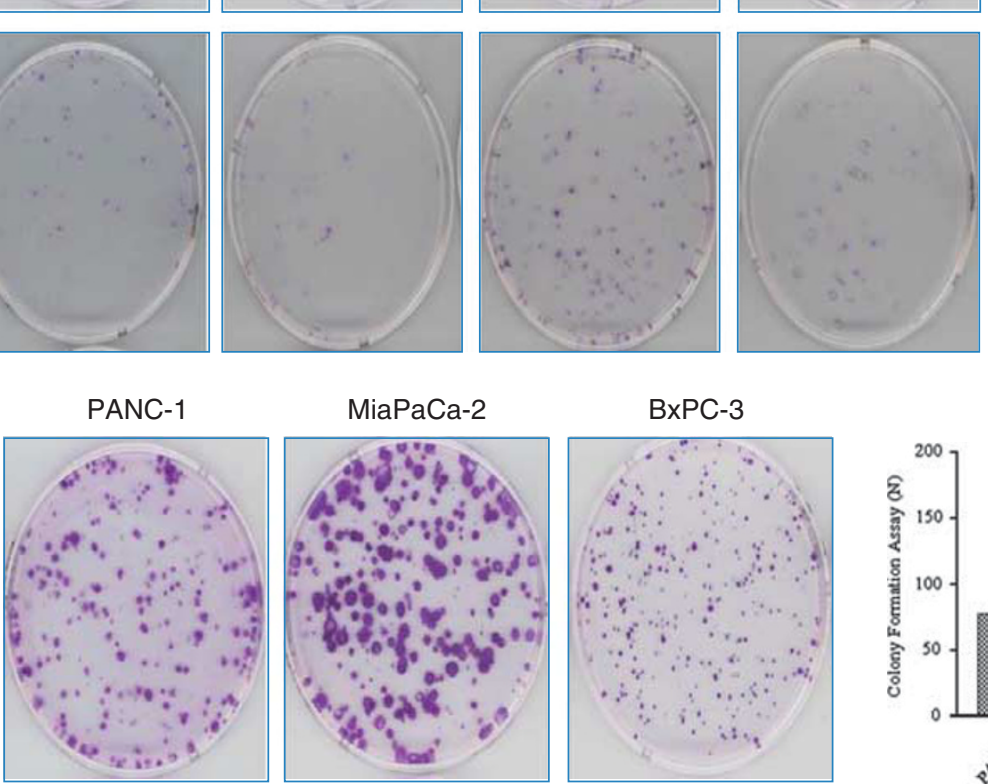

BxPC-3
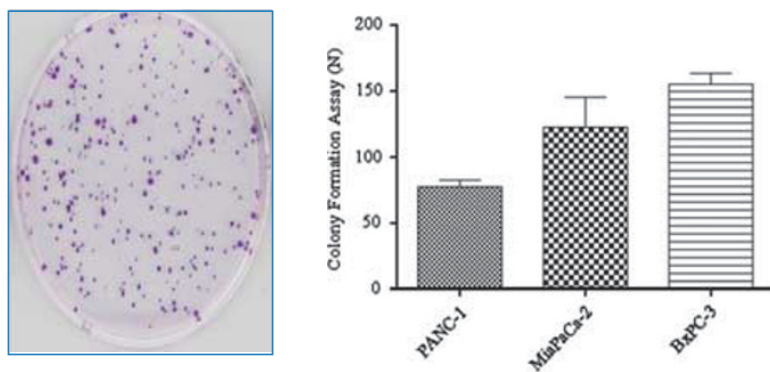

Figure 5 Colony-formation assay of MDA-PATC43, 50, 53, and 66 and control ATCC cell lines. (a) Colony-formation ability of new cell lines during earlier and later passages. (b) Colony-formation ability of commercial ATCC PDAC cell lines: PANC1, MiaPaCa-2, and BxPC-3. Cells were fixed and stained by crystal violet. Assays were done thrice and in triplicate wells. The figure is a representative of three experiments with similar results. Quantification was performed by manual counting. 
immunohistochemical analysis at the clinical core laboratory at MD Anderson. All sections were evaluated and scored by pathologists blinded to both clinical and pathologic data. Table 2 shows the details of the antibodies used. Immunohistochemical staining for E-cadherin, $\mathrm{N}$-cadherin, vimentin, CK19, $\beta$-catenin, and Smad4 was done using a Lab-Vision 480-2D immunostainer (ThermoFisher, Fremont, CA, USA). All reactions were visualized with diaminobenzidine as a chromogen. Positive and negative controls were included in each run for all the antibodies used. Isotype controls for all antibodies were negative. Two pathologists reviewed the IHC staining separately and scored each antibody expression by its percentage of area. The percentage scores were plotted by bar graph by Microsoft Excel. All images were captured via an Olympus DP72 camera attached to an Olympus BX51 microscope and processed via cellSens software.

\section{Statistical Analysis}

Statistical analyses were performed using GraphPad Prism 6.0. The two-tailed $t$ test was used for comparisons between earlier and later passages of the four new PDAC cell lines. A $P$-value of $<0.05$ was considered statistically significant.

\section{RESULTS}

Establishment, Characterization, and Validation of Early Passage Human PDAC Cell Lines

Four PDAC cell lines (MDA-PATC43, MDA-PATC50, MDAPATC53, and MDA-PATC66) were established from murine xenograft tumors of four pancreatic cancer patients. As detailed in the methods section, the cell lines were verified by our core facility to be human and unique. Figure 1 summarizes the graphic approaches in detail. Figure 2 contains representative photos of the morphology of the final estab-
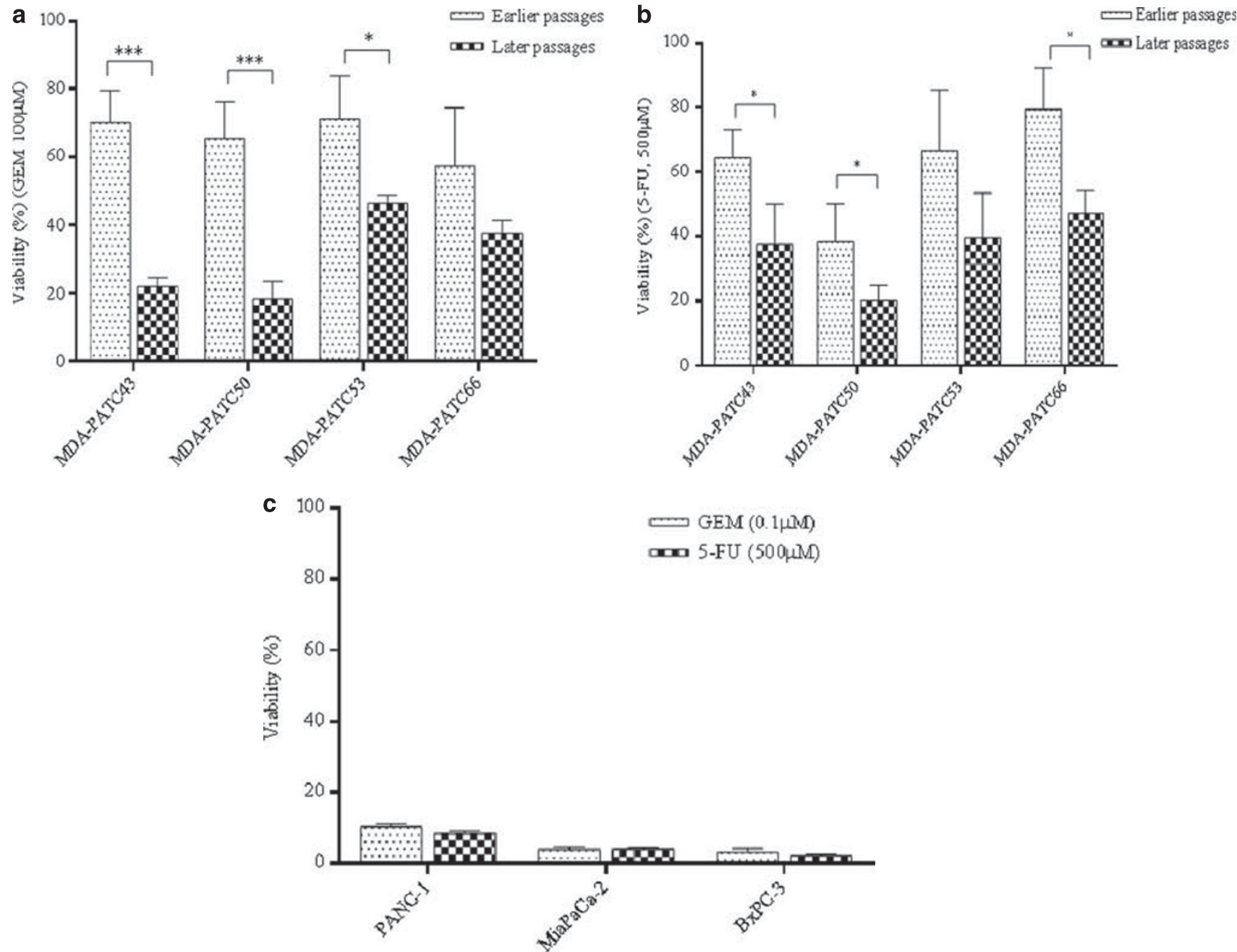

Figure 6 In vitro cytotoxicity of gemcitabine and 5-FU in newly isolated cell lines. (a) Gemcitabine and (b) 5-FU was incubated with MDA-PATC43, MDA-PATC50, MDA-PATC53, and MDA-PATC66 cells during earlier and later passages. (c) Commercial PANC-1, MiaPaCa-2, and BxPC-3 cell lines were treated with the same doses of gemcitabine and 5-FU as a control. These cells were treated for 3 days in culture, and their viability was determined with MTT assays. Assays were done thrice and in triplicate wells. Bar graphs are shown as means \pm s.d. and statistical analysis was performed by twotailed $t$ test $\left({ }^{*} P<0.05\right.$ and $\left.{ }^{* * *} P<0.001\right)$. 
lished cell lines between earlier and later passages. These PDAC cells-especially MDA-PATC53-grew as separated clumpy colonies in dishes in their early passages and as adherent monolayers with characteristic epithelial morphological features after 20 passages.

The statuses of K-ras, SMAD4, and TP53, all genes that are commonly mutated or deleted in PDAC, were evaluated via DNA or PCR exon product sequencing (Table 1). Analysis of the derived cell lines determined that SMAD4 was deleted in all cell lines except for MDA-PATC53. This was confirmed with immunohistochemical staining of SMAD4 in patients' primary tumors and corresponding PATX F1, F2, and F3 generation tumors on a tissue microarray slide (Figure 3). SMAD4 staining was strongly present in the ductal cells of Patient 53's tumor, as well as in the ductal cells in subsequent murine xenografts. SMAD4 was not present in the ductal cells of the other primary tumors or their corresponding xenografts, with the only positive staining occurring in tumor stroma.

\section{Doubling Time, Colony-Formation, and Cytotoxicity Assays}

Proliferation of these cell lines was assessed using MTT assays and their doubling times were calculated with GraphPad
Prism. The doubling times of MDA-PATC43 and MDAPATC53 did not significantly change between earlier and later passages. In the MDA-PATC50 and MDA-PATC66 cell lines, the doubling times were significantly reduced in later passages (Figures $4 \mathrm{a}, P<0.01$ and $P<0.05$, respectively). Cell cycle analysis confirmed this observation. In the MDA-PATC50 and MDA-PATC66 cell lines, the proportion of cells in the G0G1 phases increased between earlier and later passages, and the proportion of cells in the G2M checkpoint phase decreased between the earlier and later passages (Figure 4b). Cell cycle analysis of ATCC cell lines showed no significant variations in cycle phases between earlier and later passages (Figure 4c).

Colony formation was also assessed in these four new cell lines. In the cell lines MDA-PATC43, MDA-PATC50, and MDA-PATC66, colony-formation ability was significantly increased after 20 passages. Both colony size and number were significantly augmented $(P<0.01)$. Interestingly, the MDA-PATC53 cell line did not change its powerful colonyforming ability between earlier and later passages, and it grew more colonies than the other three cell lines from the start (Figures 5a and c). In ATCC cell lines, the size and number of colonies did not change significantly between subsequent passages in dishes (Figure $5 \mathrm{~b}$ and $\mathrm{d}$ ).

a
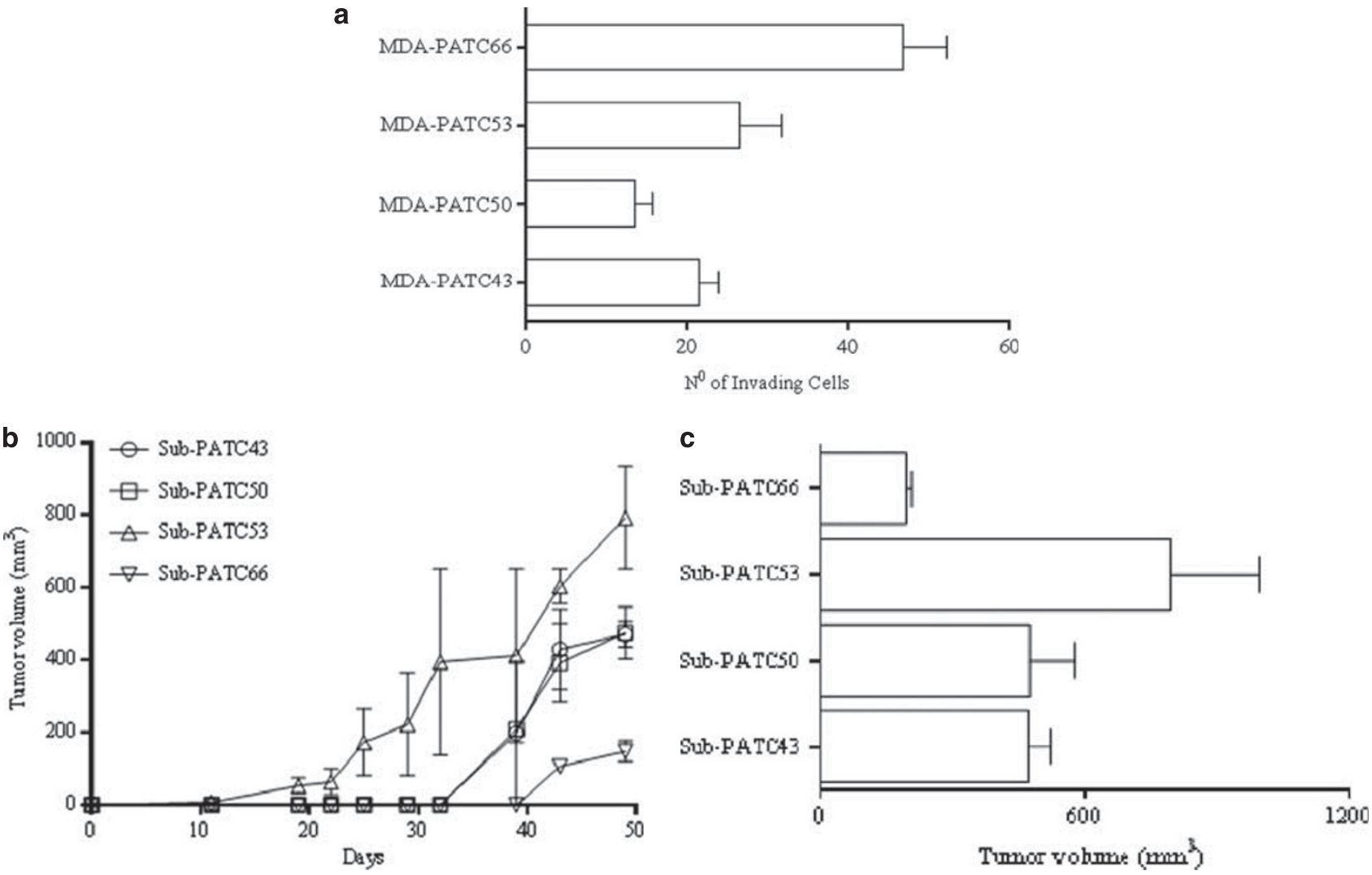

Figure 7 Invasiveness and tumorigenic nature and of new PDAC lines. (a) Cell lines' invasive capacity was determined by a standard BD BioCoat Matrigel invasion assay. Invading cells of each cell line were counted in three different fields at $\times 10$ magnification after $22 \mathrm{~h}$ of incubation. (b) All four MDA-PATC cell lines have the ability to form a progressively growing tumor after subcutaneous injection in athymic nude mice. Tumor sizes were monitored and recorded twice a week. Three mice were in each group. (c) Tumor volumes at 50 days growth. Bar graphs are shown means \pm s.d. 
Cytotoxicity assays on the four cell lines at earlier and later passages revealed that they were more sensitive to chemotherapy during later passages. The cells were treated with $100 \mu \mathrm{M}$ gemcitabine and $500 \mu \mathrm{M}$ 5-fluorouracil. The results demonstrated that MDA-PATC43, MDA-PATC50, MDA-PATC53, and MDA-PATC66 cells were more sensitive to gemcitabine in later passages $(P<0.001, P<0.001, P<0.05$, and NS, respectively, Figure 6a). Sensitivity to 5-FU also was augmented in later passages of MDA-PATC43, MDA-PATC50, and MDA-PATC66 cells significantly $(P<0.05)$ as well as in MDA-PATC53 (NS, Figure $6 \mathrm{~b}$ ). As a control, cytotoxicity assays with gemcitabine and 5-FU were also performed in PANC-1, MiaPaCa-2, and BxPC-3 cells, and sensitivity to these drugs was not altered by multiple passages in dishes (Figure 6c).

\section{In Vitro Invasiveness and In Vivo Tumorigencity}

The invasiveness of these cell lines was tested using a Boyden chamber assay and the tumorigenicity of all four new PDAC cell lines was assessed by injecting cell suspensions subcutaneously in athymic nude mice. In vitro, all cell lines had invasive capability, and MDA-PATC66 had the highest of the four (Figure 7a). In vivo, all four cell lines grew palpable tumors in 2 or 3 weeks. Interestingly, Sub-PATC53 tumors were the fastest to grow and had the largest volumes. Despite having the greatest invasiveness in vitro, MDA-PATC66 tumors were the slowest to grow and had the smallest volumes (Figures $7 \mathrm{~b}$ and c). Histology of patient tumors with corresponding PATX tumors and Sub-PATC tumors are shown in Figure 8. The histology of PATX tumors tends to more closely resemble that of the original patient tumors, with abundant stroma and duct-like structures. The Sub-PATC tumors, like other cell linederived tumors, rarely have stromal or ductal architecture resembling that of an original patient tumor, and are usually anaplastic.

\section{RPPA on PATX Tumors, PDAC Cell Lines, and Sub-PATC Tumors}

RPPA was performed with a panel of 135 protein antibodies to evaluate differences in protein expression among on PATX tumors, matched PDAC cell lines, and Sub-PATC tumors. The results of each set were normalized for protein loading a

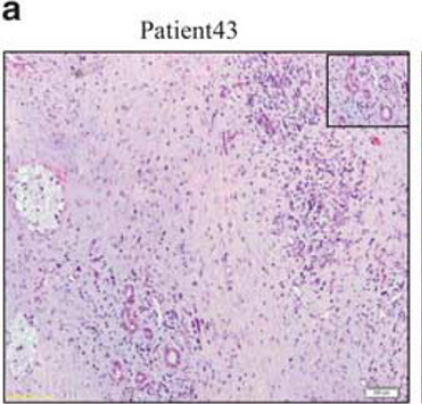

b

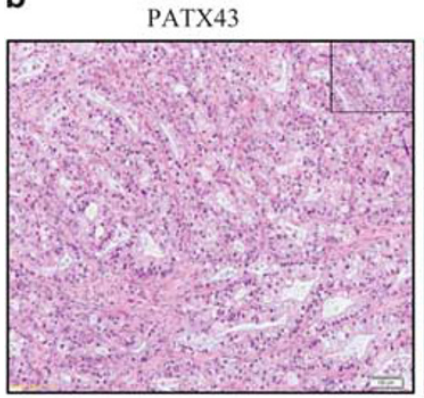

C

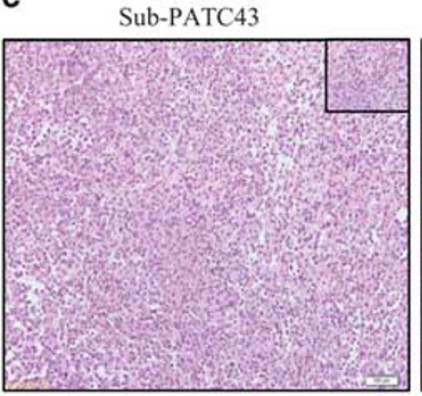

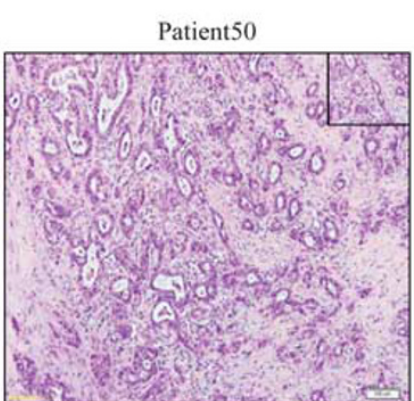

PATX50

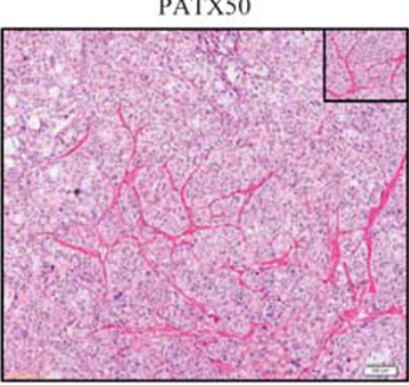

Sub-PATC50

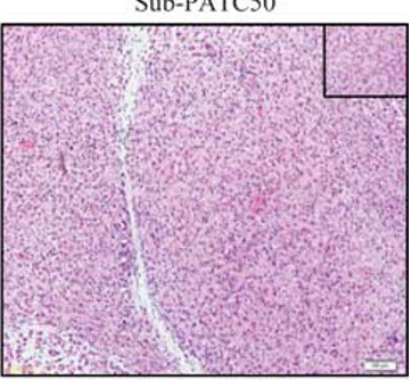

Patient53

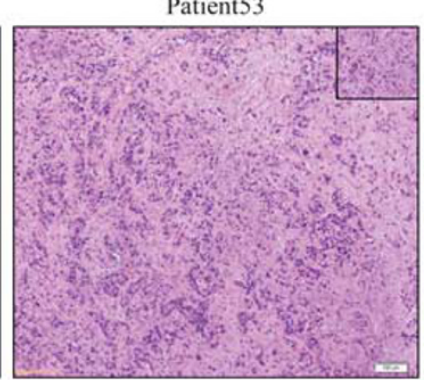

PATX53

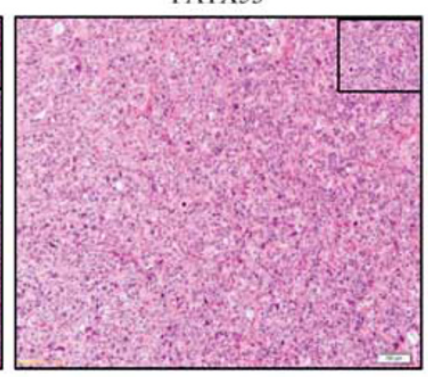

Sub-PATC53

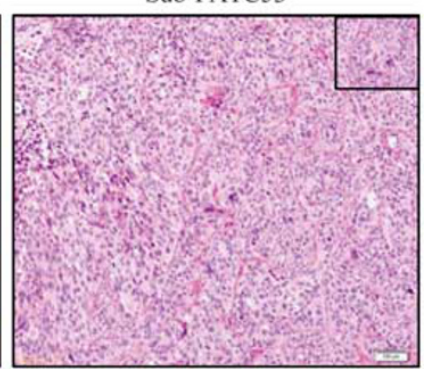

Patient66

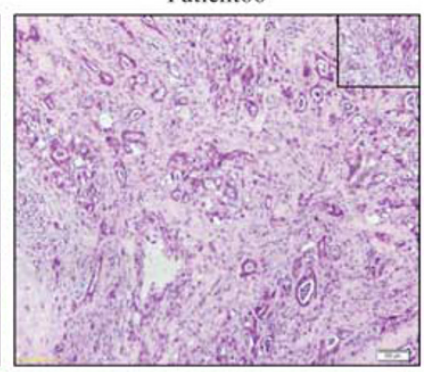

PATX66

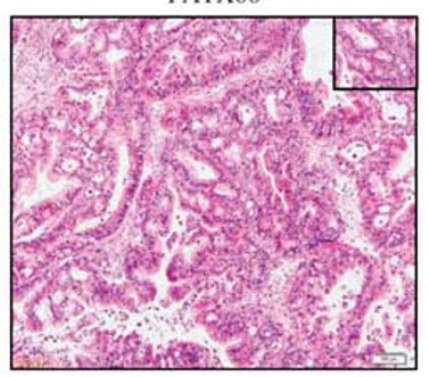

Sub-PATC66

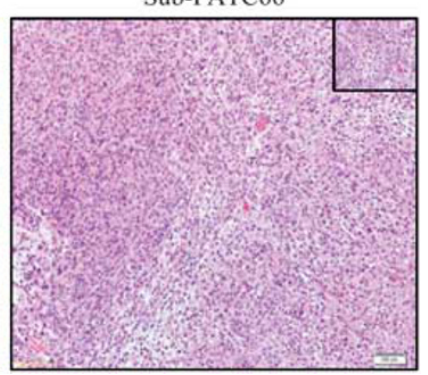

Figure 8 Representative H\&E sections of (a) patient tumor, (b) patient xenograft tumor (PATX), and (c) subcutaneous cell line tumor (Sub-PATC). PATX tumors tended to preserve some ductal architecture found in patient tumors, whereas Sub-PATC tumors tended to be anaplastic. Magnification, $\times 10$. 
a

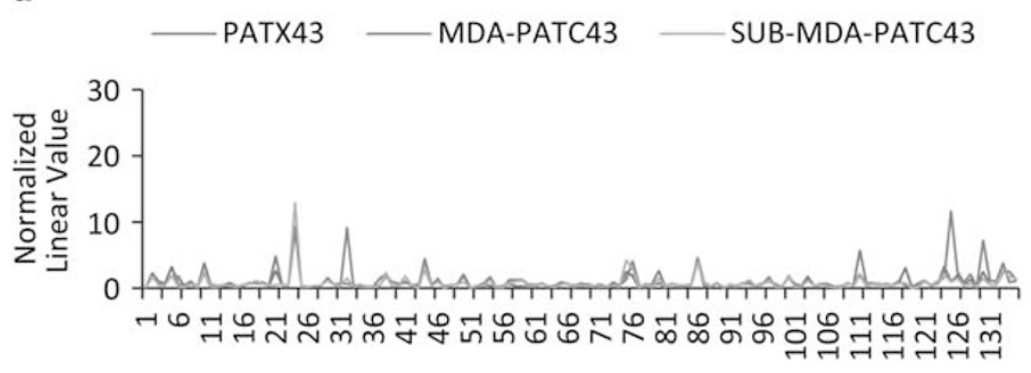

c

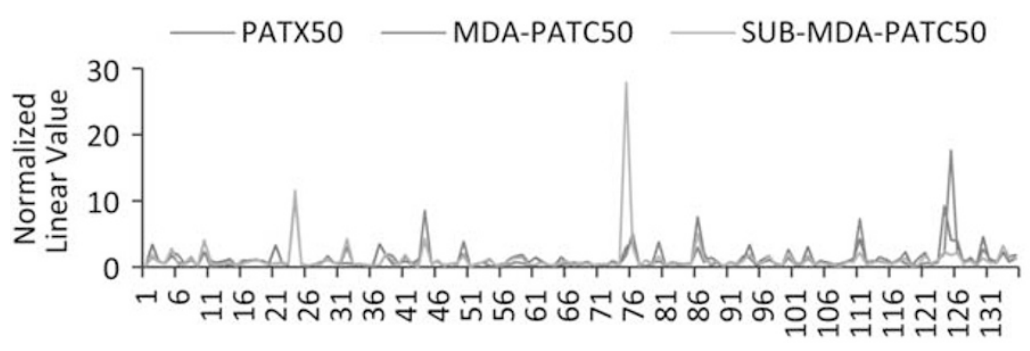

e

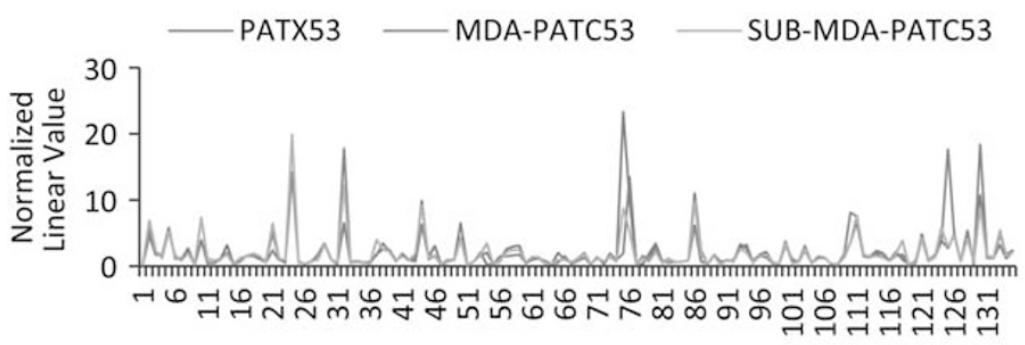

g

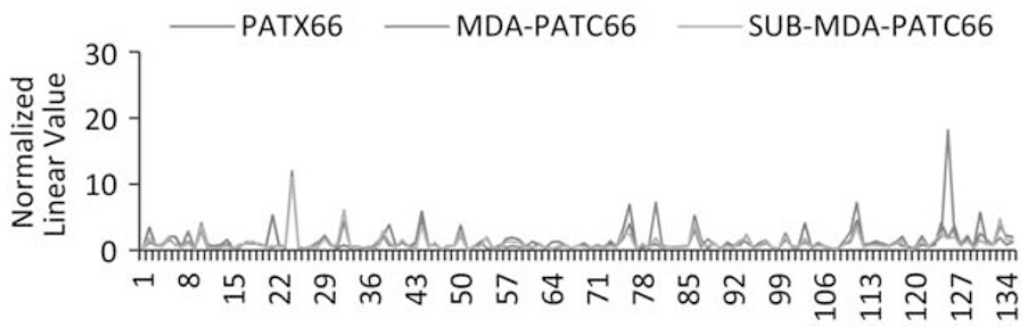

b

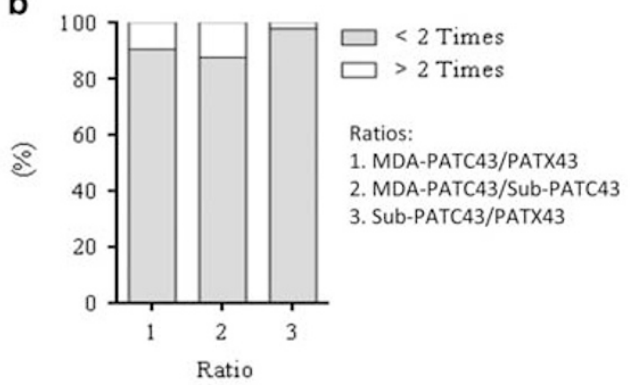

d

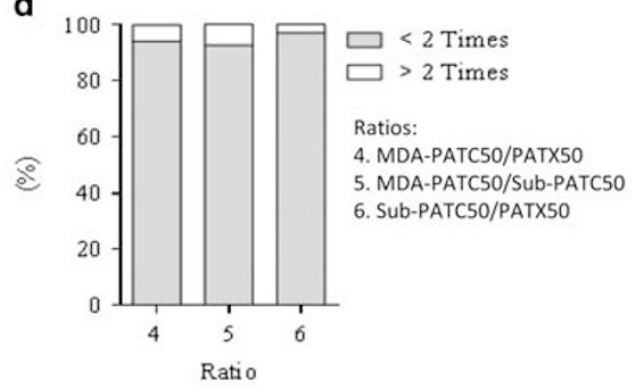

f

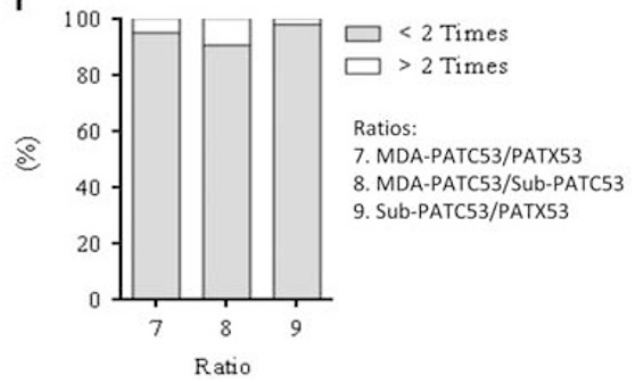

h

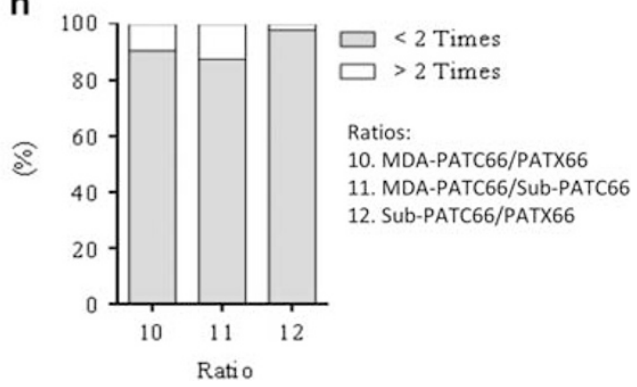

Figure 9 Proteomic concordance of patient xenograft tumors (PATX), cell lines (MDA-PATC), and cell line xenografts (Sub-PATC). (a, c, e, g) Lysates of PATX tumors, cell lines, and Sub-PATC tumors analyzed via reverse phase protein array showed close similarities in expression of most proteins. (b, $\mathbf{d}$, $\mathbf{f}$, h) Proportions of proteins expressed more or less than twofold per ratio. See Table 2 for ratio labels.

and transformed to linear values as normalized linear graphs via Microsoft Excel (Figures 9a, c, e, and g). It was evident that the pattern of protein expression among PATX tumors, PATC cell lines, and Sub-PATC tumors was generally conserved. To quantify which proteins had the most significant alterations in expression, we calculated the ratios of protein expression among all three groups and identified the proteins that had a greater than twofold change. Figure 9 (b, d, f, h) represents the proportions of proteins that were more or less than twofold in differential expression. Heatmaps of our RPPA data were generated in order to visualize patterns of protein expression among our three groups (Figure 10). These data demonstrate that differences of protein expression levels among MDA-PATC cell lines, PATX tumors, and Sub-PATC tumors was less than twofold in over $87 \%$ of the proteins on the RPPA panel. In Table 2, we listed which proteins in newly established cell lines were expressed over twofold compared with the respective xenografts from which 
a

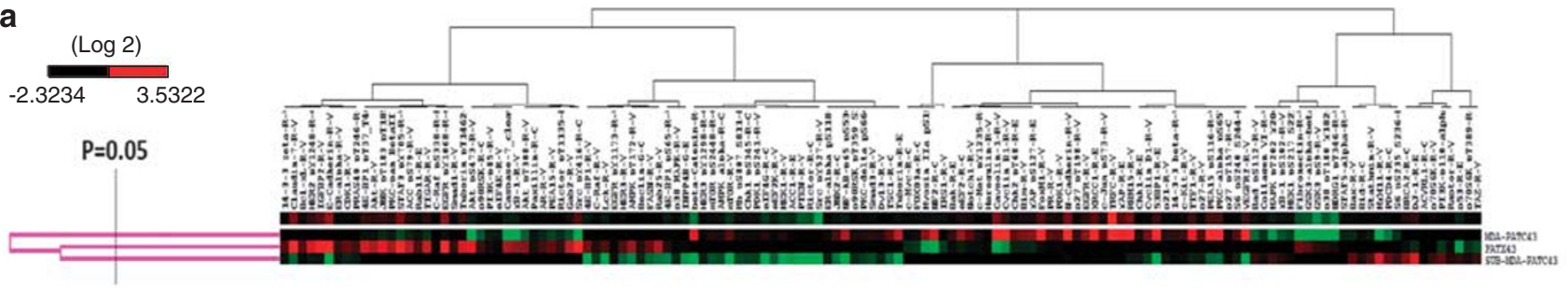

b
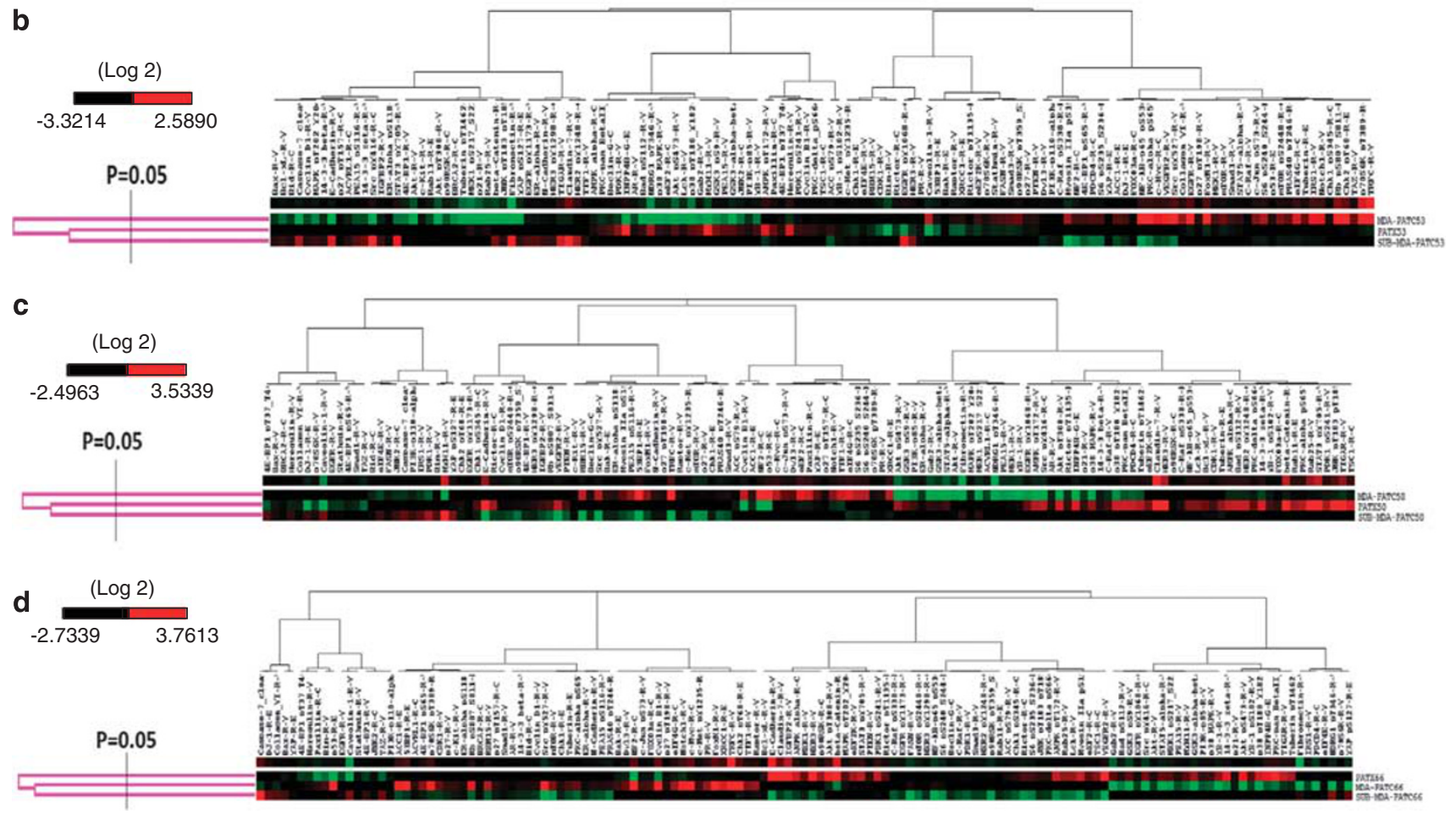

Figure 10 Unsupervised clustering heatmaps of protein expression levels in PATX tumors, MDA-PATC cell lines, and Sub-PATC tumors. These reverse phase protein array results were generated in Cluster 3.0 as a hierarchical cluster using Pearson Correlation and a center metric. The resulting heatmap was visualized in Treeview.

Table 2 List of proteins in newly established cell lines expressed over twofold compared with the xenograft tumors from which they were derived

\begin{tabular}{lll}
\hline Ratio & Proteins expressed over twofold & \% of total \\
MDA-PATC43/PATX43 & Caveolin1; Cyclin B1; FoxM1; Myosin-Ila-pS1943; N-Cadherin; NF2; PEA15-pS116; & \\
& PKC-alpha-pS657; RBM15; TFRC; VEGFR2; XRCC1; YAP & \\
MDA-PATC50/PATX50 & Caveolin1; Cyclin-B1; eEF2; NF2; p53; TFRC; TTF1 & 9.6 \\
MDA-PATC53/PATX53 & Caveolin1; FoxM1; NFkB.p65-pS536; p70S6K-pT389; Rb-pS807; Src-pY527; TFRC \\
MDA-PATC66/PATX66 & ACC1; Caveolin1; Chk2; Cyclin B1; FoxM1; NF2; Notch1; p53; p70S6K; SrC; TFRC & 5.9 \\
\hline
\end{tabular}

they were derived. The cell lines overexpressed a number of proteins in common, including Caveolin-1, NF2, FoxM1, Cyclin-B1, and the Transferrin receptor (TFRC). The relative expression of these proteins was secondarily evaluated with western blot analysis (Figure 11b). In MDA-PATC43 and 50,
Caveolin-1 slightly decreased with passage number. In MDAPATC66, Caveolin-1 expression continued to increase with passages. MDA-PATC53, which was derived from a liver metastasis of a primary PDAC, retained high Caveolin-1 expression in its xenograft and all throughout in vitro 
a

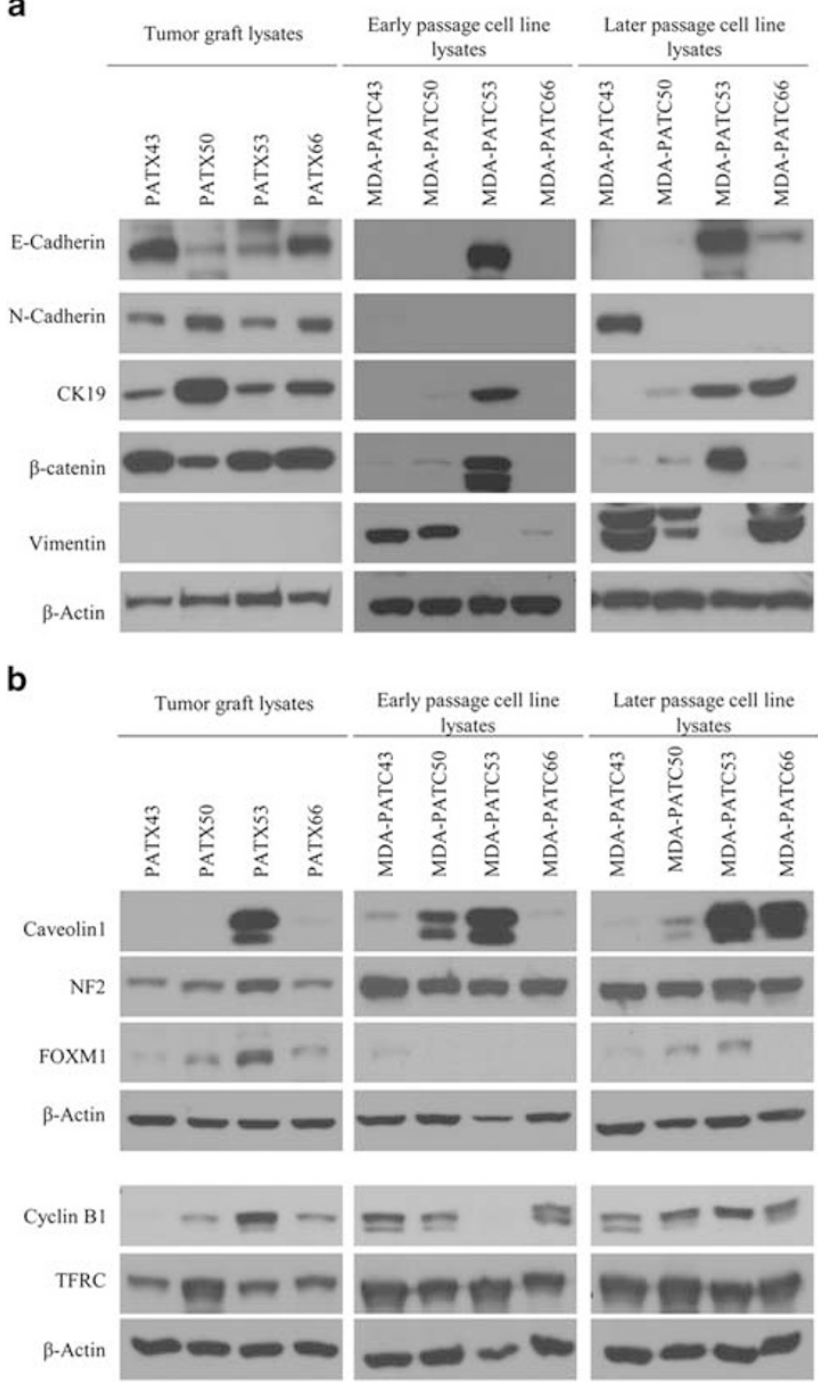

Figure 11 Western blot comparison of PATX tumors with early and later passage cell lines. (a) Relative protein expression levels of the epithelial and mesenchymal markers $\mathrm{E}$-cadherin, $\mathrm{N}$-cadherin, vimentin, cytokeratin19 , and $\beta$-catenin. $\beta$-Actin was used as the loading control. MDA-PATC43 showed increased expression of N-cadherin and vimentin. MDA-PATC50 showed increased expression of CK19 and vimentin. MDA-PATC53 maintained high expression of E-cadherin, $\mathrm{CK} 19$, and $\beta$-catenin. MDAPATC 66 showed increased expression of E-cadherin, CK19, and vimentin. (b) The relative protein expression levels of caveolin-1, NF2, FoxM1, cyclin $B 1$, and transferrin receptor (TFRC). These were proteins found to be upregulated at least twofold on cell lines compared with the PATX tumors they were derived from, based on RPPA data (see Table 2). Caveolin-1, NF2, and TFRC were generally increased in cell lines compared with PATX tumors. FoxM1 decreased in early passage cell lines but then was re-expressed in later cell lines. Cyclin B1 was lost in early passage MDA-PATC53, but was re-expressed in later passages, whereas the three other cell lines continued to increase expression compared with PATX tumors.

passages. NF2 expression was increased in all cell lines compared with their respective xenografts. FoxM1 decreased in early passage cell lines but then was re-expressed in later cell lines, with the exception of MDA-PATC66. Cyclin-B1 was lost in early passage MDA-PATC53, but was re-expressed in later passages, whereas the three other cell lines continued to increase expression compared with PATX tumors. TFRC expression was increased in all cell lines compared with PATX tumors.

\section{EMT Marker Variation Between In Vitro and In Vivo Tumor Cell Growth}

EMT-related markers were compared in cell lysates of MDA-PATC during earlier $(<5)$ passages and later $(>20)$ passages via western blot analysis. N-cadherin expression was increased in later passages of MDA-PATC43. CK19 expression was increased in later passages of MDA-PATC50 and MDA-PATC66. Vimentin expression was substantially escalated in later passages of MDA-PATC43, MDA-PATC50, and MDA-PATC66 (Figure 11a). These western blot results suggest that MDA-PATC43, MDA-PATC50, and MDA-PATC66 were transformed to the mesenchymal phenotype during cultivation in dishes. Interestingly, MDA-PATC53 sustained its epithelial phenotype throughout earlier and later passages. It consistently maintained high expression of E-cadherin and $\beta$-catenin. IHC staining of E-cadherin, $\mathrm{N}$-cadherin, vimentin, CK19, and $\beta$-catenin was performed in primary patient tissues with matched PATX and sub-PATC tissues (Figures 12 and 13). Slides were scored based on positive staining of the tumor cells only, not the stromal cells. IHC staining scores showed that the level of E-cadherin expression between primary and PATX tumors was retained on PATX43 and PATX66 xenografts, and decreased in PATX50 and PATX53 ( $80 \%$ to $60 \%, 70 \%$ to $40 \%$, respectively). Staining scores of E-cadherin were decreased dramatically to 10 and $5 \%$ on all sub-PATC tumors, suggesting loss of the epithelial cell phenotype when the cell lines were grown in vivo (Figures 12a and c). Staining scores of $\mathrm{N}$-cadherin expression between primary and PATX tumors demonstrated a different pattern. $\mathrm{N}$-cadherin staining decreased from the patient tumor to the PATX tumor in PATX43 and PATX53, whereas N-cadherin staining increased in PATX50 and PATX66. However, in all four groups, N-cadherin increased substantially in the SubPATC tumors, which suggested a transition to the mesenchymal phenotype during in vivo tumorigenesis (Figures $12 \mathrm{~b}$ and c). Vimentin staining, although weak on patient tumors and PATX tumors, was intense on Sub-PATC43, 50, and 66. However, it remained negligible on all Patient 53 samples (Figure 13a). CK19 staining was augmented on PATX43, PATX50, and PATX53 compared with primary tumors, and reduced on sub-PATC43, 50, and 66. It is worth noting that CK19 staining remained relatively high throughout all Patient 53 samples (Figure 13b). High $\beta$-catenin staining intensity was preserved from all patient tumors to PATX tumors, and was subsequently diminished in Sub-PATC43, 50, and 66, yet remained high in Sub-PATC53 (Figure 13c). These results verified our findings that MDA-PATC43, 50, and 66 cell lines were transformed from epithelial to mesenchymal states during their processing both in vitro and 

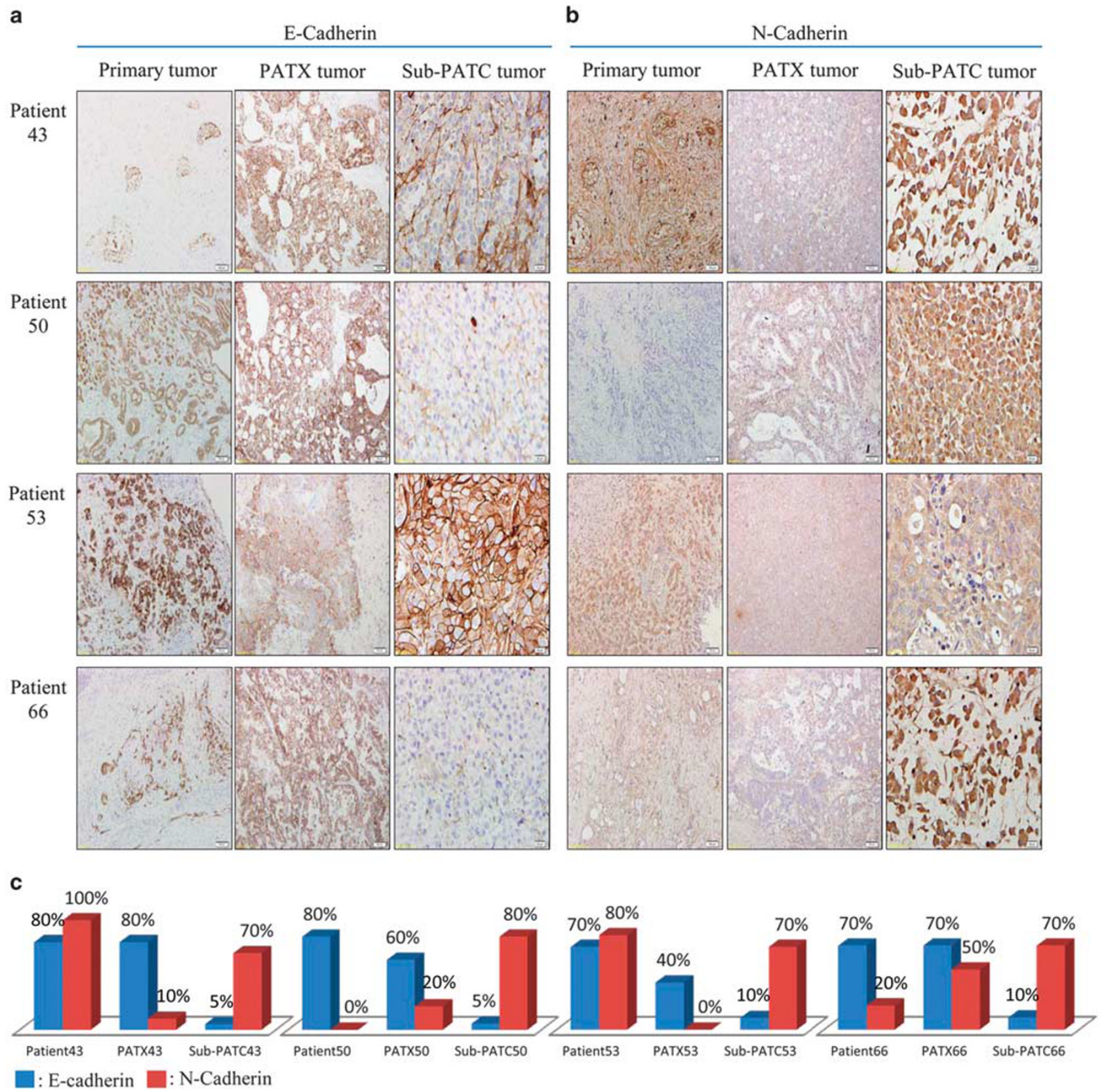

Figure 12 Immunohistochemical evaluation of EMT-related markers in primary patient tumors, PATX tumors, and Sub-PATC tumors. (a) E-cadherin and (b) N-cadherin expression on tumor cells was evaluated. (c) Percentages of positive tumor cells per slide were quantified by pathologists. E-cadherin persisted in patient and PATX tumors, but was weak in Sub-PATC tumors. N-cadherin was variably expressed in patient and PATX tumors, but significantly increased in Sub-PATC tumors. Magnification, $\times 10$.

in vivo, and curiously MDA-PATC53, which was derived from a liver metastasis site of primary PDAC, preserved its strongly epithelial phenotype in the process of cultivation and tumorigenicity.

\section{DISCUSSION}

We report the establishment and characterization of four new PDAC cell lines: MDA-PATC43, MDA-PATC50, MDA-PATC53, and MDA-PATC66, which were derived from patient tumor murine xenografts. Early passage and well-characterized human PDAC cell lines are important resources for studying cancer cell biology, as well as for developing new strategies against cancer cell growth and new drug therapy validation in vivo and in vitro. ${ }^{19}$ The first report on successful cultivation of a human PDAC was in $1963,{ }^{20}$ and since then, numerous PDAC cell lines have been reported and characterized, ${ }^{21}$ but little is known about how these cell lines change over time in culture. Most of the previously 
a

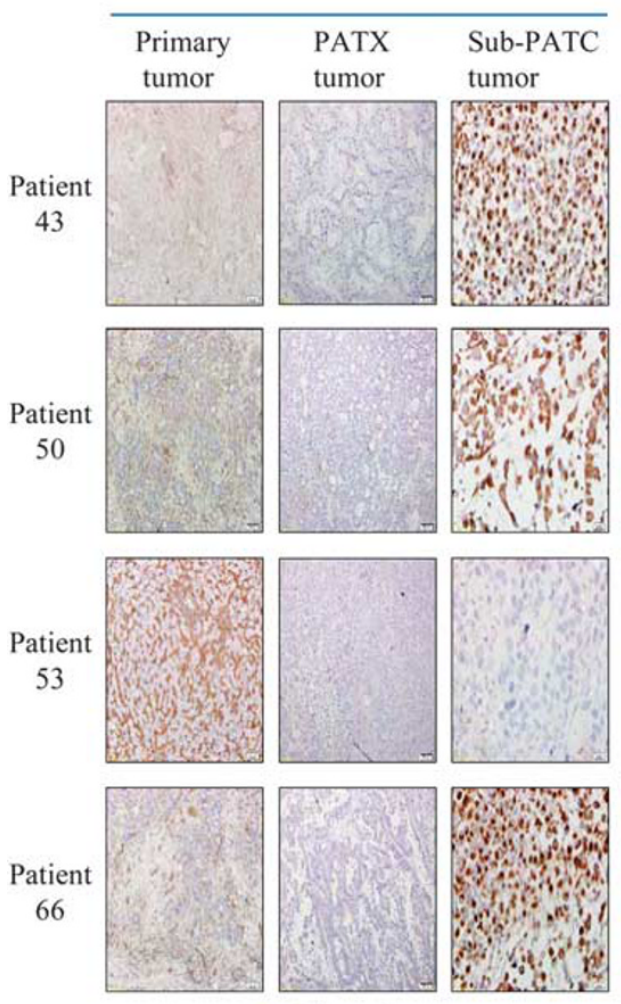

- Vimentin

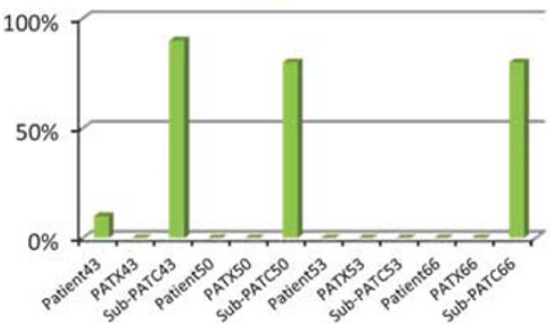

b
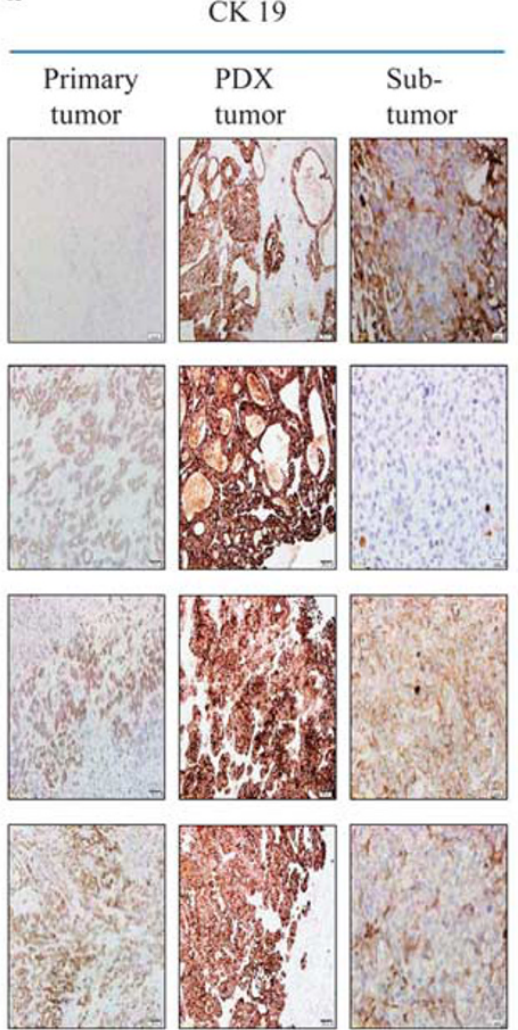

C

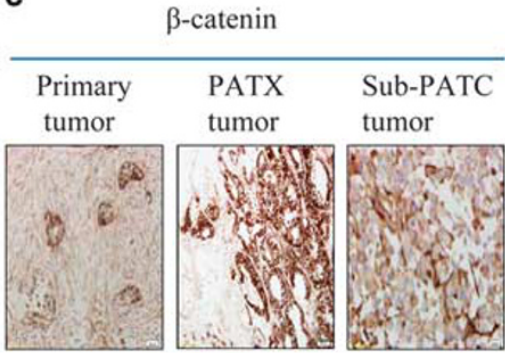
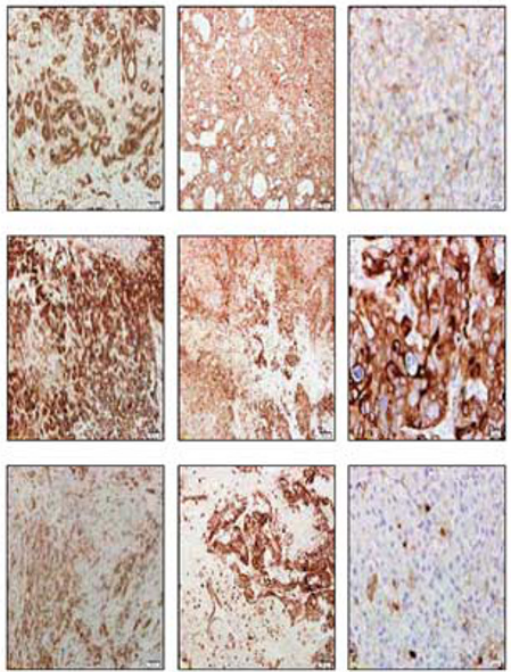

- $\beta$-Catenin

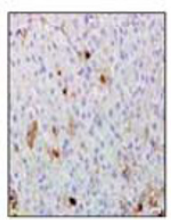

지 19

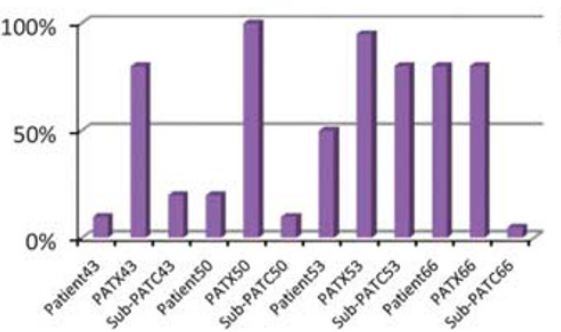

Figure 13 Further immunohistochemical evaluation of EMT-related markers in primary patient tumors, PATX tumors, and Sub-PATC tumors. Expression of (a) vimentin, (b) cytokeratin-19, and (c) $\beta$-catenin was evaluated. The lowest panel displays percentages of positive tumor cells per slide as determined by pathologists. For Patients 43, 50, and 66, vimentin was negligible in patient and PATX tumors, but became strongly expressed in SubPATC tumors. CK19 was highly expressed in PATX tumors, but substantially decreased in Sub-PATC tumors. $\beta$-Catenin staining was also intense in PATX tumors, and significantly weaker in Sub-PATC tumors. For Patient 53, all three tumor types showed little vimentin and strong expression of CK19 and $\beta$-catenin. Magnification $\times 10$.

established PDAC cell lines used in research were derived from primary pancreatic tumors, tumor metastases, or ascitic effusions, whereas all of our new cell lines were derived from murine patient tumor xenografts. We have found this approach to be beneficial because of the relatively larger amounts of tumor tissue from which to culture cells, as primary patient tumor specimens are usually more limited in volume. Also, murine xenografts are potentially endlessly expandable, so it is possible to isolate new cell lines long after the original tumor resection has taken place. The four PDAC cell lines we established have demonstrated efficient tumorigenicity when injected subcutaneously into nude mice, with a success rate of $100 \%$ (data not shown). Therefore, this approach can be used to produce durable cell lines that are able to be experimented with both in vitro and in vivo. This model enables concurrent study of patient tumor xenografts and their derived cell lines, which creates a robust platform to compare the biology of cancer cells living confined in mouse stromal tissues to cancer cells living as pure populations.

In this study, we have also shown that PDAC cells from a primary pancreatic tumor site take on a predominately mesenchymal phenotype when isolated from murine xenograft tumors, and this mesenchymal phenotype is further augmented when these cell lines are grown as subcutaneous 
tumors in immunocompromised mice. On the other hand, one cell line, MDA-PATC53, isolated from a liver metastasis of a pancreatic primary adenocarcinoma, maintained a predominately epithelial phenotype. Also, MDA-PATC53 maintained a wild-type SMAD4 gene from the initial surgical resection, to xenograft establishment, and finally to cell line isolation. This suggests that epithelial or mesenchymal phenotypic changes in culture may depend on the tissue of origin and whether the cells came from a primary tumor $v s$ a metastasis. Future studies will be required to further delineate the changes in cancer cell biology that occur upon establishment of cell lines from patient or xenograft tissues, and how their tumor environments of origin affect these changes. This information can potentially be used to help reconcile experimental findings in vitro and in vivo, in order to overcome limitations often seen when therapies are translated from the petri dish to animal models and finally to human patients.

\section{ACKNOWLEDGMENTS}

This work was funded by Viragh Family Foundation and Various Donors in Pancreatic Cancer Research, and was supported by Cancer Center Support Grant (CCSG) core resources of Flow Cytometry and Cellular Imaging Facility, Functional Proteomics Reverse Phase Protein Array (RPPA) Core, and Research Animal Support Facility-Houston under $\mathrm{NIH} / \mathrm{NCl}$ award (P30CA016672). STR DNA fingerprinting was done by the Cancer Center Support Grant-funded Characterized Cell Line core is funded by $\mathrm{NCl}$ \#CA016672. This work was supported in part by National Institutes of Health (NIH) grant T32CA009599 (DR).

\section{DISCLOSURE/CONFLICT OF INTEREST}

The authors declare no conflict of interest.

1. Howlader N, Noone AM, Krapcho M, et al. (eds). SEER Cancer Statistics Review, 1975-2010, Bethesda, MD, http://seer.cancer.gov/csr/1975 2010/based on November 2012 SEER data submission, posted to the SEER web site, 2013.

2. Jemal A, Siegel R, Ward E, et al. Cancer statistics, 2008. CA Cancer J Clin 2008;58:71-96.

3. Douglas EJ, Fiegler $\mathrm{H}$, Rowan A, et al. Array comparative genomic hybridization analysis of colorectal cancer cell lines and primary carcinomas. Cancer Res 2004;64:4817-4825.

4. Larramendy ML, Lushnikova T, Bjorkqvist AM, et al. Comparative genomic hybridization reveals complex genetic changes in primary breast cancer tumors and their cell lines. Cancer Genet Cytogenet 2000;119:132-138.

5. Chaffer $\mathrm{CL}$, Weinberg RA. A perspective on cancer cell metastasis. Science 2011;331:1559-1564.

6. Huntsman DG, Caldas $\mathrm{C}$. Assignment1 of the E-cadherin gene $(\mathrm{CDH} 1)$ to chromosome 16q22.1 by radiation hybrid mapping. Cytogenet Cell Genet 1998;83:82-83.

7. Besco JA, Hooft van Huijsduijnen R, Frostholm A, et al. Intracellular substrates of brain-enriched receptor protein tyrosine phosphatase rho (RPTPrho/PTPRT). Brain Res 2006;1116:50-57.

8. Thiery JP, Acloque $\mathrm{H}$, Huang RY, et al. Epithelial-mesenchymal transitions in development and disease. Cell 2009;139:871-890.

9. Yachida S, Zhong Y, Patrascu R, et al. Establishment and characterization of a new cell line, A99, from a primary small cell carcinoma of the pancreas. Pancreas 2011;40:905-910.

10. Hotz HG, Hines OJ, Foitzik $\mathrm{T}$, et al. Animal models of exocrine pancreatic cancer. Int J Colorectal Dis 2000;15:136-143.

11. Metildi CA, Kaushal S, Hoffman RM, et al. In vivo serial selection of human pancreatic cancer cells in orthotopic mouse models produces high metastatic variants irrespective of Kras status. J Surg Res 2013;184: 290-298.

12. Kim MP, Evans DB, Wang $\mathrm{H}$, et al. Generation of orthotopic and heterotopic human pancreatic cancer xenografts in immunodeficient mice. Nature Protoc 2009;4:1670-1680.

13. Ding Z, Wu CJ, Chu GC, et al. SMAD4-dependent barrier constrains prostate cancer growth and metastatic progression. Nature 2011; 470:269-273.

14. Liu Y, Bodmer WF. Analysis of P53 mutations and their expression in 56 colorectal cancer cell lines. Proc Natl Acad Sci USA 2006;103: 976-981.

15. Chang Z, Li Z, Wang X, et al. Deciphering the mechanisms of tumorigenesis in human pancreatic ductal epithelial cells. Clinical Cancer Res 2013;19:549-559.

16. Sheehan KM, Calvert VS, Kay EW, et al. Use of reverse phase protein microarrays and reference standard development for molecular network analysis of metastatic ovarian carcinoma. Mol Cell Proteomics 2005;4:346-355.

17. Paweletz CP, Charboneau L, Bichsel VE, et al. Reverse phase protein microarrays which capture disease progression show activation of prosurvival pathways at the cancer invasion front. Oncogene 2001;20: 1981-1989.

18. Li Z, Chang Z, Chiao LJ, et al. TrkBT1 induces liver metastasis of pancreatic cancer cells by sequestering Rho GDP dissociation inhibitor and promoting RhoA activation. Cancer Res 2009;69:7851-7859.

19. Philip PA, Mooney $M$, Jaffe $D$, et al. Consensus report of the national cancer institute clinical trials planning meeting on pancreas cancer treatment. J Clin Oncol 2009;27:5660-5669.

20. Dobrynin YV. Establishment and characteristics of cell strains from some epithelial tumors of human origin. J Natl Cancer Inst 1963;31: 1173-1195.

21. Deer EL, Gonzalez-Hernandez J, Coursen JD, et al. Phenotype and genotype of pancreatic cancer cell lines. Pancreas 2010;39:425-435. 